\title{
Fabriken zum Zeitvertreib? - Illustrierte Zeitschriften
}

Mit dem starken Wirtschaftswachstum der 189oer Jahre setzte sich auch im Zarenreich der Massenkonsum durch. Besonders in den russischen Städten veränderte er das Alltagsleben und die Freizeit der Menschen. Doch auch in ländlichen Gebieten wandelten sich die Konsumpraktiken der Bevölkerung. ${ }^{1}$ Gleichzeitig entwickelte sich eine populäre Massenkultur, zu der mit Fotografien illustrierte Zeitschriften gehörten. Sie erfreuten sich nach der Jahrhundertwende im ganzen Zarenreich großer Beliebtheit. Voraussetzung für die Entstehung dieser Publikationen waren technische Innovationen wie die der Rotationsdruckmaschine. Nun konnten Druckereien in kürzester Zeit hohen Auflagen produzieren. ${ }^{2}$ Um 1900 verbreitete sich im Zarenreich ebenfalls der Rasterdruck, dank dem Fotografien als kostengünstige Autotypien in Printmedien erscheinen konnten. ${ }^{3}$ Beide Neuerungen sorgten dafür, dass die allgemeinen Druckkosten zurückgingen und immer mehr Menschen Zugang $\mathrm{zu}$ russischen Illustrierten erhielten. Kostengünstige, bebilderte Zeitschriften spielten gerade im vorrevolutionären Russland eine wichtige Rolle, weil es im Vergleich zu anderen Westeuropäischen Ländern viele Menschen gab, die nicht lesen konnten. ${ }^{4}$ Der relativ geringe Textanteil und die zahlreichen Abbildungen ermöglichten auch ihnen, an der Massenkultur Anteil zu nehmen. ${ }^{5}$

1 Catriona Kelly; Steve Smith: Commercial Culture and Consumerism, in: Catriona Kelly; David Shepherd (Hrsg.): Construction Russian Culture in the Age of Revolution, 1881-1940, Oxford, New York 1998, S. 106-164, S. 130.

2 Die Rotationsdruckmaschinen konnten in der Stunde ca. 3.00o Ausgaben der Zeitschrift Niva drucken. Glinternik: Reklama v Rossii, S. 76.

3 Zur Rolle der Fotografie in der Presse siehe: Bernd Weise: Der zögerliche Einsatz von Fotos in der Tagespresse deutscher Verlage. Korrekturanmerkungen zur Geschichtsschreibung des Fotojournalismus, in: Ulrich Hägele; Irene Ziehe (Hrsg.): Gedruckte Fotografie. Abbildung, Objekt und mediales Format, Münster 2015, S. 13-30, S. 14-18.

4 In ländlichen Gebieten konnten in den 19ioer Jahren nur etwa 25 Prozent der männlichen Bevölkerung lesen und schreiben. Allerdings war dieser Bevölkerungsanteil in industrialisierten Gebieten sehr viel höher. Brooks: When Russia Learned to Read, S. 4; Richard Pipes: Die russische Revolution, Bd. 3. Rußland unter dem neuen Regime, Berlin 1993, S. 529.

5 Botrel untersucht diesen Prozess für die spanische Bevölkerung, die einen ähnlich niedrigen Alphabetisierungsgrad hatte. Jean-François Botrel: Entre imprimé et oralité. Léssort de la culture de masse en Espagne (1833-1936), in: Jean-Yves Mollier; Jean-François Sirinelli; 
Russische Redakteure propagierten in den Blättern das Lesen von Zeitungen und Zeitschriften als eine neue Form der Freizeitbeschäftigung. ${ }^{6}$ Dementsprechend verfolgte die Illustrierte Presse um die Jahrhundertwende in erster Linie das Ziel, ihre Leser zu unterhalten. ${ }^{7}$ Die Quellengrundlage dieses Kapitels bilden sechs illustrierte Zeitschriften, deren Artikel sich besonders dem Kulturleben, der Politik sowie dem Sport widmeten. Die Redakteure orientierten sich an der urbanen Lebenswelt der beiden russischen Metropolen, in denen die Redaktionen ihren Sitz hatten. ${ }^{8}$ St. Petersburg und Moskau waren Anfang des 20. Jahrhunderts nicht nur kulturelle Zentren, sondern auch wichtige Industriestandorte. In beiden Städten prägten die Spuren der Industrialisierung das Stadtbild und waren für die Mehrheit der Leser tagtäglich präsent. Frank Bösch und Friedrich Lenger betonen in ihren Untersuchungen zu Printmedien um die Jahrhundertwende, dass sich die neue Massenpresse besonders durch ihren lokalen Bezug auszeichnete. ${ }^{9}$ Dieser Annahme folgend wird in diesem Kapitel die Frage aufgeworfen, welche Rolle russische Journalisten in ihren Berichten aus dem Alltag der Großstädte der Industrie zubilligten, welchen Unterhaltungswert die Redaktionen russischen Fabriken zuschrieben.

Wer neben dem Herausgeber und den Redakteuren nahm Einfluss auf die thematische Ausrichtung der Zeitschriften? Zunächst waren Illustrierte Konsumprodukte, die sich an ein bestimmtes Publikum richteten..$^{10}$ Die Leser trugen an die Zeitschriften ihre Erwartungshaltungen heran und sahen vom Kauf ab, wenn der Inhalt des Blattes nicht ihren Vorstellungen entsprach.

François Vallotton (Hrsg.): Culture de masse et culture médiatique en Europe et dans les Amériques 1860-1940, Paris 2006, S. 143-156, S. 146.

6 West: I Shop in Moscow, S. 111. Hierzu auch: James von Geldern; Louise McReynolds (Hrsg.): Entertaining Tsarist Russia. Tales, Songs, Plays, Movies, Jokes, Ads, and Images from Russian Urban Life, 1779-1917, Bloomington 1998.

7 Holzer untersucht dieses Phänomen besonders für die österreichische und die deutsche Presse, doch seine Forschungsergebnisse treffen auch auf das Zarenreich zu. Anton Holzer: Rasende Reporter. Eine Kulturgeschichte des Fotojournalismus. Fotografie, Presse und Gesellschaft in Österreich 1890 bis 1945, Darmstadt 2014, S. 11.

8 In erster Linie wandten sich Journalisten mit ihren Texten auch an eine Leserschaft in diesen beiden Städten. Stolarski: The Rise of Photojournalism, S. 76. Ein Teil der hier präsentierten Forschungsergebnisse wurde bereits in folgendem Artikel veröffentlicht: Lenka Fehrenbach: Vom Randthema zum patriotischen Vorzeigemotiv. Industriefotografie in russischen Illustrierten vor 1917, in: Fotogeschichte. Beiträge zur Geschichte und Ästhetik der Fotografie, Jg. 34/2015, Heft 136, S. 25-32.

$9 \quad$ Frank Bösch: Mediengeschichte. Vom asiatischen Buchdruck zum Fernsehen, Frankfurt a. M., New York 2011, S. 112; Lenger: Metropolen der Moderne, S. 228.

10 Gilles Feyel: L'économie de la presse au XIX siècle, in: Dominique Kalifa u. a. (Hrsg.): La civilisation du journal. Histoire culturelle et littéraire de la presse française au XIX $\mathrm{X}^{\mathrm{e}}$ siècle, Paris 2011, S. 141-180, S. 141. 
Folglich orientierten sich die Herausgeber mit den Themen an den Vorlieben ihrer Kunden. Darüber hinaus nahm im ausgehenden 19. und beginnenden 20. Jahrhundert die Politik mit Zensurmaßnahmen Einfluss auf die Inhalte der russischen Presse." Und schließlich versuchten auch außerstaatliche Akteure, beispielsweise Fabrikanten, bestimmte Inhalte in den Zeitungen zu platzieren. ${ }^{12}$ Aus dieser Gemengelage mehr oder weniger gezielter Themensetzungen und kommerzieller Abwägungen entstanden thematische Trends in den Medien.

In Anlehnung an Niklas Luhmann zeigt sich jedoch, dass diese Aufmerksamkeitszyklen für einen Gegenstand nicht mit der öffentlichen Meinung gleichgesetzt werden dürfen. ${ }^{13}$ Die Redakteure trugen zunächst nur dazu bei, bestimmte Inhalte anzusprechen und dadurch die Diskussion und das Interesse der Menschen zu wecken. Auf dieser Annahme basierend verfolgt die Analyse russischer Illustrierter das Ziel zu zeigen, wie die Herausgeber der Zeitschriften zur Industrialisierung standen und wie sie das Interesse ihrer Leser einschätzten. Dies ist deshalb aufschlussreich, weil die illustrierte Presse es einer breiten Öffentlichkeit ermöglichen konnte, sich mit Fotografien aus Fabriken einen Eindruck von diesen ihnen sonst verschlossenen Orten zu verschaffen. ${ }^{14}$

Die Untersuchung beschränkt sich nicht allein auf Fotografien von und Artikel über Firmen, sondern nimmt auch die breiteren gesellschaftlichen Auswirkungen der Industrialisierung in den Blick, beispielweise das enorme Wachstum der russischen Städte. ${ }^{15}$ Indem die Industrie immer mehr Arbeitskräfte benötigte, strömten zunehmend Menschen aus ländlichen Gebieten in die urbanen Zentren. Auf diese Explosion der Einwohnerzahlen waren die

11 Zur wechselseitigen Beziehung zwischen Politik und Medien allgemein: Georg Rudinger: Medienmacht und Massenwirkung. Von der fragmentierten Öffentlichkeit zur internationalen Kommunikationsgesellschaft, in: Ulrich Lappenküper; Joachim Scholtyseck; Christoph Studt (Hrsg.): Masse und Macht im 19. und 20. Jahrhundert. Studie zu Schlüsselbegriffen unserer Zeit, München 2003, S. 127-144, S. 128.

12 Anlässlich von Jubiläen bemühten sich beispielsweise Unternehmer, Meldungen über die Festlichkeiten und über Glückwünsche prominenter Persönlichkeiten in der Presse zu platzieren. Siehe: Kapitel „Inszenierte Geschichte - Firmenjubiläen“, S. 179-240.

13 Niklas Luhmann: Öffentliche Meinung, in: Politische Vierteljahresschrift, Jg. 11/1970, Heft 1, S. 2-28, zu Aufmerksamkeitsregeln insbesondere S. 10-11.

14 Sowohl Firmenalben als auch Festschriften richteten sich in erster Linie an ein exklusives Publikum. Siehe: Kapitel „Neue Bilder eines neuen Raums - Fabrikalben“ und „Inszenierte Geschichte - Firmenjubiläen“.

15 Mitte des 19. Jahrhunderts lebten in der russischen Hauptstadt etwa 485.00o Menschen, in Moskau etwa 337.00o. In den folgenden 30 Jahren wuchsen die Einwohnerzahlen um mehr als das Doppelte an, so dass in den 188oer Jahren beide zu Millionenstädten wurden. 1915 hatten St. Petersburg mit 2.318.6oo und Moskau mit 1.984.00o die Anzahl ihrer Bewohner ein weiteres Mal in etwa verdoppelt. Colton: Moscow, S. $757-758$. 
Städte nicht vorbereitet, Mangel an Wohnraum ${ }^{16}$ und schlechte hygienische Lebensbedingungen ${ }^{17}$ waren die Folgen. Im vorliegenden Kapitel wird untersucht, ob Journalisten über diese Themen in Verbindung mit Fabriken berichteten und möglicherweise Kritik formulierten. Die Analyse gibt Hinweise darauf, was die Redakteure ihren Rezipienten zumuteten, ohne dass die Blätter ihren Unterhaltungscharakter einbüßten. ${ }^{18}$ Schließlich fungierten Illustrierte auch als Werbeträger für Handelsgüter und damit als Multiplikatoren des Massenkonsums, weshalb abschließend analysiert wird, wie Unternehmer die Reklameteile der Zeitschriften als Plattform nutzten, um ihre eigenen Produkte anzupreisen.

Grundlage für die Analyse sind die sechs illustrierten Zeitschriften Iskry (Die Funken), Niva (Das Feld), Ogonëk (Flämmchen), Solnce Rossii (Sonne Russlands), weiter Ženskoe Delo (Frauenangelegenheit) sowie die illustrierte Beilage der Tageszeitung Novoe Vremja (Neue Zeit). Ausgewertet wurden jeweils die kompletten Jahrgänge der Illustrierten ab dem Jahr 1898 beziehungsweise der Ersterscheinung. 1898 bildet deshalb das Anfangsdatum, weil russische Illustrierte zu diesem Zeitpunkt vermehrt begannen, Fotografien abzudrucken. Den Endpunkt des Untersuchungszeitraums stellt der Beginn des Ersten Weltkriegs 1914 dar. ${ }^{19}$

Die Analyse der sechs Blätter ergab einen Untersuchungskorpus von ca. 90 bebilderten Artikeln und Bildserien (ca. 220 Autotypien), die Fabriken thematisieren. Die Studie wird ergänzt durch Texte und Fotografien, die sich mit den Folgen der Industrialisierung auseinandersetzen, aber häufig nur indirekt auf die Industrie verweisen. Zwar zählen diese Bilder nicht zur

16 Ruble: Second Metropolis, S. 266; Goehrke: Auf dem Weg in die Moderne, S. 317; Engel: Between the Fields and the City; Ekaterina Slanskaia: House Calls. A Day in the Practice of a Duma Woman Doctor in St. Petersburg, in: Toby W. Clyman; Judith Vowles (Hrsg.): Russia through Women's Eyes. Autobiographies from Tsarist Russia, New Haven 1996, S. $186-216$.

17 Lee: Das Volk von Moskau und seine bedrohte Gesundheit, S. 16-17, 34; Martin: Der Schmutz, der Gestank und die Stadt, S. 363-364. Zu Pocken-, Typhus- und Choleraepidemien in Moskau siehe auch: Manfred Späth: Wasserleitung und Kanalisation in Grossstädten. Ein Beispiel der Organisation technischen Wandels im vorrevolutionären Russland, in: Forschungen zur osteuropäischen Geschichte, Bd. 25, Berlin 1978, S. 342360, S. 343 .

18 McReynolds betont, dass über das Freizeitverhalten wichtige Rückschlüsse darüber gezogen werden können, wie sich die Gesellschaft den großen Veränderungen des ausgehenden 19. Jahrhunderts stellte und wodurch sie sich in ihrer Selbstdefinition auszeichnete. Louise McReynolds: Russia at Play. Leisure Activities at the End of the Tsarist Empire, Ithaca, London 2003, S. 3-4.

19 Die Analyse der Berichterstattung während des Ersten Weltkriegs findet sich im Kapitel „Die Fabrik wird salonfähig - der Erste Weltkrieg“, S. 325-368. 
Industriefotografie, weil sie weder in Fabriken entstanden, noch Fabriken als Motiv zeigen, sie sind aber Teil des Kontexts, in dem Fabrikfotografien in Zeitschriften erschienen.

Für die Untersuchung der Zeitschrifteninhalte wurden zunächst alle Titel und Bilder tabellarisch erfasst. Die Tabelle führt das Datum, die Zeitschrift, den Industriezweig und den grographischen Ort der Fabrik auf. Notiert wurde ferner die Länge des Berichts, und ob es sich um eine Fotografie mit Begleittext handelt, eine Produktfotografie, Portraits oder Gruppenaufnahmen sowie ob die Aufnahmen Menschen zeigen. Weiter hält die Tabelle fest, in welchem Kontext (Jubiläum, Unglück, Reklame etc.) der Bericht entstand. Ausgehend von dieser Auswertung wurden thematische Gruppen erstellt und einer detaillierteren Analyse unterzogen. ${ }^{20}$

Leider ist die Quellenlage zum Entstehungsprozess der Zeitschriften schwierig. Alle hier untersuchten Blätter mussten ihr Erscheinen einstellen, nachdem die Bolschewiki 1917 an die Macht gekommen waren. Die Redaktionsarchive, sofern sie vorhanden waren, sind nicht erhalten. Vereinzelt finden sich in den Unterlagen offizieller Behörden Hinweise zu den Illustrierten. ${ }^{21}$ Diese beschränken sich auf formale Fragen zur inhaltlichen Ausrichtung der Zeitschrift. Über die Arbeitsweise in den Redaktionen oder auf die Frage, welche Kriterien über die Publikation bestimmter Fotografien entschieden, geben sie keine Antworten.

\subsection{Bilder informieren das Zarenreich}

Die Entstehung des Journalismus und die Publikation der ersten Zeitung fielen im Zarenreich in die Regierungszeit Peters I. um die Wende vom 17. zum 18. Jahrhundert. ${ }^{22}$ Russische Zeitschriften erschienen ab dem ersten Drittel des 18. Jahrhunderts und präsentierten schon bald Holzschnitte oder Kupferstiche auf den Titelseiten. ${ }^{23}$ Illustrationen blieben lange ein Luxus, weshalb Leser sie

20 Zur Methode der qualitativen Bildanalyse siehe: Kapitel „Neue Bilder eines neuen Raums - Fabrikalben“, S. 103-178.

21 Beispielweise: die Kanceljarii Glavnago Upravlenija po delam pečati (Hauptkanzlei des Amtes für Druckangelegenheiten) zu Ogonëk: RGIA f. 776, op. 17, d. 28; oder zu Iskry: RGIA f. 776, op. 8, d. 1361.

22 Anna Kask; Marina Mochnačeva: Vvedenie, in: Marina Mochnačeva (Hrsg.): Russkij illjustrirovannyj žurnal, 1703-1941, Moskva 2006, S. 4-35, S. 4.

23 Hierzu auch: Gary Marker: Publishing, Printing, and the Origins of Intellectual Life in Russia. 1700-1800, Princeton 1985, S. 97. 
als eigenständige Bilder sammelten. ${ }^{24}$ Auch mit dem Aufkommen der Fotografie blieben Stiche das am weitesten verbreitete visuelle Medium in Zeitschriften. Fotografische Aufnahmen bekamen zunächst nur indirekt Zugang zu den Publikationen, indem sie als Vorlage für die Arbeit der Holzschneider dienten. ${ }^{25}$

Als eigenständige Illustrationen erschienen gedruckte Fotografien erst um 1900. ${ }^{26}$ Damit hatte Russland etwa zehn Jahre Rückstand auf Printmedien in Deutschland oder in den Vereinigten Staaten. ${ }^{27}$ Die russische Presselandschaft griff die neue Technik des Halbtonverfahrens auf. Die so hergestellten Autotypien waren anfangs noch teuer, dennoch übernahmen russische Herausgeber sie bald und entwickelten das Druckverfahren auf eigene Kosten so lange weiter, bis sich der Preis für Illustrationen verringerte. Mit Verbreitung der Rotationspressen stieg die Anzahl der Publikationen bis zum Ersten Weltkrieg stark an. Habbo Knoch und Daniel Morat bezeichnen die Periode ab den 1880er Jahren als „massenmediale Sattelzeit“, als Phase der Verdichtungs- und Beschleunigungsprozesse. ${ }^{28}$ Neben den technische Neuerungen waren es, wie Frank Bösch bemerkt, besonders staatliche Maßnahmen der Lockerung oder Aufhebung der Zensur, die zu einem starken Pressewachstum führten. ${ }^{29}$ Erste Schritte in diese Richtung fanden in Russland im Zuge der Großen Reformen unter Alexander II. statt. Eine Reaktion hierauf war 1866 die Gründung der ersten russischen Nachrichtenagentur Rossijskoe Telegrafnoe Agenstvo

24 Kask; Mochnačjova: Vvedenie, S. 5-8, 10.

25 Bildunterschriften vermerkten teilweise explizit, wenn die Vorlage eines Stichs eine Fotografie gewesen war. Beispielsweise: Sostojanie rabot na Mastinskom mostu, utrom 31 Dekabrja 1869 g., in: Vsemirnaja Illjustracija, 10.01.1870, S. 40. Zum Herstellungsverfahren von Holzstichen siehe: Gerry Beegan: The Mass Image. A Social History of Photomechanical Reproduction in Victorian London, New York 2008, S. 174, 177; Paul Hogarth: The Artist as Reporter, London 1986, S. 30-31.

26 Bereits etwa 20 Jahre früher wäre ihr Druck technisch möglich gewesen, doch westeuropäische Zeitungen hatten erst in den $1890 e r$ Jahren begonnen, die neue Druckweise finanziell zu fördern, und erst, als das Verfahren kommerziell gewinnbringend war, übernahmen die Redaktionen die Technik. Holzer: Rasende Reporter, S. 53. Eine Übersicht weiterer Drucktechniken findet sich in: Benson (Hrsg.): The Printed Picture, S. 218-230.

27 Beegan: The Mass Image, S. 160; Elena Chernevich: Commercial Graphics, in: Michail Ankist; Nina Burina (Hrsg.): Russian Graphic Design, London 1990, S. 87-92, S. 90-91; Bernd Weise: Pressefotografie. I. Die Anfänge in Deutschland. Ausgehend von einer Kritik bisheriger Forschungsansätze, in: Fotogeschichte, Jg. 31/1989, Heft 9, S. 15-40, S. $20-21$.

28 Habbo Knoch; Daniel Morat: Medienwandel und Gesellschaftsbilder 1880-1960. Zur historischen Kommunikologie der massenmedialen Sattelzeit, in: dies. (Hrsg.): Kommunikation als Beobachtung. Medienwandel und Gesellschaftsbilder 1880-1960, München 2003, S. 9-33, S. 19-20.

29 Dies galt neben Russland auch für Frankreich oder Deutschland. Bösch: Mediengeschichte, S. 110-111. 
(Russische Telegraphenanstalt), mittels derer russische Zeitungen Zugang zu ausländischen Informationen bekamen. ${ }^{30}$ Die Zensurbehörden kontrollierten weiterhin die Inhalte der Presse, was bei der Interpretation der Illustrierten beachtet werden muss. 1906 erfolgte die zweite große Ausweitung der Pressefreiheit, als die russische Regierung die Vorzensur aufhob. ${ }^{31}$ Nun durften Minister nicht mehr willkürlich Texte oder ganze Veröffentlichungen verbieten, nur ein Gerichtsurteil konnte diese Maßnahme anordnen. ${ }^{32}$ Diese Liberalisierung führte vor dem Ersten Weltkrieg zu vielen Neugründungen von Zeitungen, Zeitschriften und Illustrierten. Im Jahr 1914 konnten russische Leser zwischen ca. 3.00o Zeitschriften und Illustrierten wählen. ${ }^{33}$

Entscheidend für die wachsende Zahl russischer Pressepublikationen waren neben der liberaleren Pressepolitik der zunehmende Alphabetisierungsgrad: Um die Jahrhundertwende war regelmäßiges Zeitunglesen kein Privileg der Oberschichten mehr. ${ }^{34}$ In ländlichen Gebieten lasen die Menschen meist Texte, von denen sie sich einen praktischen Nutzen versprachen. Beispielsweise erhofften sich die Leser währen des Russisch-Japanischen Kriegs (19041905) Informationen über den Konflikt zu erhalten. ${ }^{35}$

In den Städten waren die wöchentlich erscheinenden Illustrierten der beliebteste Lesestoff der einfachen Bevölkerungsschichten. ${ }^{36}$ Im Zarenreich unterteilten sich illustrierte Zeitschriften in zwei Gruppen: diejenigen, die

30 Owen Johnson: The Roots of Journalism in Central and Eastern Europa, in: Jerome Aumente u. a. (Hrsg.): Eastern Europe Journalism. Before, During and After Communism, Cresskill 1999, S. 5-40, S. 14.

31 Charles Ruud: Fighting Words. Imperial Censorship and the Russian Press, 1804-1906, London ${ }^{2} 2009$, S. 207, 225. Leider ist keine detaillierte Auflistung derjenigen Themen überliefert, welche unter die Zensur fielen. Svetlana Jakovlevna Machonina: Istorija russkoj žurnalistiki načala XX veka. Učebnoe posobie, Moskva 2002, S. 17-25. Zur Geschichte der russischen Zensur allgemein: P. S. Rejfman: Iz istorii russkoj, sovetskoj i postsovetskoj cenzury, URL: http://reifman.ru/russ-tsenzura/ (zuletzt eingesehen am 21.04.2017); P. S. Rejfman: Cenzura v dorevoljucionnoj, sovetskoj i postsovetskoj Rossii, Tom 1, Cenzura v dorevoljucionnoj Rossii, Moskva 2015.

32 Jacob Walkin: Government Controls Over the Press in Russia, 1905-1914, in: Russian Review, Jg. 13/1954, Heft 3, S. 203-209, S. 203.

33 Allerdings führten Materialkürzungen während des Kriegs dazu, dass viele Publikationen ihr Erscheinen einstellten. Kask; Mochnačjova: Vvedenie, S. 33.

34 Brooks: When Russia Learned to Read, S. 27-28.

35 Corinne Gaudin: Circulation and Production of News and Rumor in Rural Russia during World War I, in: Murray Frame u. a. (Hrsg.): Russian Culture in War and Revolution, 1914-22. Book 2. Political Culture, Identities, Mentalities, and Memory, Bloomington 2014, S. $55^{-71}$, S. 56 .

36 Auf dem Land sahen viele durch das Lesen geistlicher Literatur die Möglichkeit, ihre Seele zu retten. Arbeiter hingegen hatten einen weiteren Horizont und griffen zu sehr unterschiedlichen Genres. Brooks: When Russia Learned to Read, S. 31-33, 111. 
eigenständige Publikationen waren, und diejenigen, die gegen einen kleinen Aufpreis zumeist am Wochenende als Zusatz zu etablierten Zeitungen erschienen.

Die Illustrierte Niva war um die Jahrhundertwende die bedeutendste selbstständige Zeitschrift und erschien von 1870 bis 1917 in St. Petersburg. Der deutsche Herausgeber Adolph F. Marks (1838-1904) hatte die Familienzeitschrift 1868 gegründet. Um 1900, zu Beginn des Untersuchungszeitraums, war sie mit einer Auflagenzahl von ca. 200.00o Stück die beliebteste russische Zeitschrift. ${ }^{37}$ Sie richtete sich an eine städtische, gebildetere Leserschaft, Kaufleute, Lehrer und Priester, aber auch Arbeiter griffen zu dieser Lektüre. $^{38}$ Viele Herausgeber von Illustrierten, die um die Jahrhundertwende neue Blätter gründeten, orientierten sich am Layout und Inhalt der Niva. Mit der zunehmenden Konkurrenz verlor die Zeitschrift bis zum Ersten Weltkrieg ihre Vorreiterrolle. ${ }^{39}$ Inhaltliche Schwerpunkte waren russische Kunst und Literatur, Geschichte sowie Meldungen über aktuelle Ereignisse. Die zeitnahe visuelle Berichterstattung zu aktuellen Geschehnissen stellte eine der großen Neuerungen der illustrierten Zeitschriften dar. Zur Zeit der Holz- und Kupferstiche dauerte die Herstellung der Bilder so lange, dass sie erst mehrere Wochen später erscheinen konnten. ${ }^{40}$

Zum Untersuchungskorpus zählt weiter Solnce Rossii, die von 1912 bis 1917 als großformatige Publikation in St. Petersburg erschien. ${ }^{41}$ Die aufwendig gestaltete und auf dickes Papier gedruckte Zeitschrift widmete sich Themen wie Literatur, Theater und zeitgenössischer Kunst. Sie richtete sich an die gehobene Mittelschicht und war sowohl Statussymbol als auch Informationsquelle. ${ }^{42}$

Die Illustrierte Ženskoe Delo wandte sich speziell an eine weibliche Leserschaft. Sie wurde in Moskau in den Jahren 1910 bis 1917 herausgegeben. Die Redaktion präsentierte ihren Leserinnen neben Artikeln über Feminismus auch Beiträge zu Mode oder Kinderbetreuung. ${ }^{43}$

Ogonëk erschien 1899 zunächst als Beilage zur liberalen Tageszeitung Birževye Vedomosti (Börsenmeldungen) in St. Petersburg. Der Schwerpunkt

37 Brooks: When Russia Learned to Read, S. 111-113.

38 Borodkin; Chugunov: The Reading Culture of Russian Workers, S. 159.

39 Stolarski: The Rise of Photojournalism, S. 53-55.

40 Stolarski: The Rise of Photojournalism, S. 20.

41 Marina P. Mochnačeva (Hrsg.): Russkij illjustrirovannyj žurnal. 1703-1941, Moskva 2006, S. 313-323.

42 Stolarski: The Rise of Photojournalism, S. 61-63.

43 June Pachute Farris: Sources for the Study of Russian Women Journalists. A Bibliographic Essay, in: Barbara Norton; Jehanne Gheith (Hrsg.): An Improper Profession. Women, Gender, and Journalism in Late Imperial Russia, Durham, London 2001, S. 249-279, S. 270. 
der Zeitung lag auf Wirtschaftsthemen. ${ }^{44}$ Die Beilage widmete sich hingegen Berichten aus Wissenschaft, Literatur, Kunst und Politik. ${ }^{45}$ Sie wandte sich an ein Publikum mit geringer Bildung, war auf billiges Papier gedruckt und deutlich günstiger als Niva. ${ }^{46} \mathrm{Ab} 1908$ bis 1918 erschien Ogonëk als eigenständige Zeitschrift, die sich besonders auf aktuelle Ereignisse im In- und Ausland konzentrierte. Sowohl das Papier, als auch die gedruckten Fotografien der Illustrierten hatten eine bessere Qualität und machten das Blatt sehr populär. Nach eigenen Angaben stieg die Auflage von ca. 100.000 Exemplaren im Jahr 1910 auf 700.000 Ende 1914. ${ }^{47}$ Ogonëk war die Zeitschrift mit der höchsten Auflage im Zarenreich.

Ebenfalls in St. Petersburg kam die konservative und regierungsnahe Tageszeitung Novoe vremja heraus. ${ }^{48}$ Die von Zar Nikolaus II. bevorzugte Zeitung richtete sich an ein gebildetes Publikum. 1899 bis 1917 erschien einmal wöchentlich die gleichnamige illustrierte Beilage, die ebenfalls Teil des analysierten Quellenkorpus ist. ${ }^{49}$ Das Blatt profitierte maßgeblich davon, dass Aleksej Sergeevič Suvorin (1834-1912), einer ihrer Herausgeber, Lizenzen für Kioske an 44 der 6o Eisenbahnstrecken im Zarenreich erwarb. Hier ließ Suvorin Drucksachen wie Zeitschriften, aber auch Postkarten aus der eigenen Produktion an Reisende verkaufen. ${ }^{50}$

Die letzte Illustrierte des Quellenkorpus ist die Beilage der eher liberalen Tageszeitung Russkoe Slovo (Das Russische Wort), ${ }^{51}$ die von 1901 bis 1917 unter

44 L. N. Beljaev; M. K. Zinov'ev; M. M. Nikiforov (Hrsg.): Bibliografija periodičeskich izdanij Rossii 1901-1916, 2. Bd., Leningrad 1959, S. 513; Louise McReynolds: The News under Russia's Old Regime. The Development of a Mass-Circulation Press, Princeton 1991, S. 3, 131,283 .

45 RGIA f. 776 , op. 17 , d. 28, l. 2.

46 Ein Jahresabonnement von Niva kostete in St. Petersburg 5,5 Rubel, während Leser von Ogonëk nur 2 Rubel im Jahr zahlen mussten. Brooks: When Russia Learned to Read, S. 111113; RGIA f. 776 , op. 17, d. 28, l. 2 .

47 o. A.: Otkryta podpiska na 1915 god, in: Ogonëk, 25.12.1914, S. 17.

48 Zum Zeitpunkt ihrer Gründung war die Novoe Vremja ein liberales Blatt, orientierte sich aber nach der Übernahme durch Suvorin stark nach rechts. Edvard Radzinsky: Alexander II. The Last Great Tsar, New York 2005, S. 339.

49 Wortman: Scenarios of Power, Bd. 2, S. 494; McReynolds: The News under Russia's Old Regime, S. 25; Mary Zirin: Meeting the Challenge. Russian Women Reporters and the Balkan Crises, in: Barbara Norton, Jehanne Gheith (Hg.): An Improper Profession. Women, Gender, and Journalism in Late Imperial Russia, Durham, London 2001, S. 140-166, S. 141.

50 Machonina: Istorija russkoj žurnalistiki načala XX veka, S. 177 .

511895 gegründet, sollte Russkoe Slovo nach dem Wunsch seines Herausgebers Sytin eine Brücke zwischen Kulturschaffenden und Bevölkerung bilden. McReynolds: The News under Russia's Old Regime, S. 168-169, 216, 240, 256. 
dem Namen Iskry in Moskau erschien. ${ }^{52}$ Nach mehrmonatigen Verhandlungen mit den russischen Behörden hatte Ivan Dmitrievič Sytin (1851-1935) die Genehmigung erhalten, eine Publikation zu drucken, ${ }^{53}$ welche Themen wie Literatur, gesellschaftliche Ereignisse, Kunst und Sport umfasste. ${ }^{54}$ Mit dieser Konzeption orientierte sich die Zeitschrift inhaltlich an den weltweit ersten Illustrierten, der Illustrated London News und L'Illustration aus Paris. ${ }^{55}$ Sie richtete sich an Leser aller Gesellschaftsgruppen und publizierte neben einer Vielzahl an Fotografien auch Karikaturen, Witze und populäre Lieder. Damit deckte sie ein breiteres Themenspektrum ab als beispielsweise Niva. ${ }^{56}$

Heute lässt sich kaum mehr nachvollziehen, wie die Illustrierten ihren Bildbedarf deckten. Hinweise auf die Autoren geben manchmal die Bildunterschriften. Teilweise vermerkten sie die Initialen der Fotografen, in anderen Fällen hielten sie fest, dass ein Fotograf ihrer Zeitschrift das Bild gemacht habe. ${ }^{57}$ Manche Blätter wählten auch die Formulierung: „für die Zeitschrift ${ }^{45}$ - im Auftrag der Zeitschrift. Dies beschreibt die Situation besser, denn Lichtbildner arbeiteten nie ausschließlich für ein Blatt. Der Beruf des Pressefotografen existierte um die Jahrhundertwende noch nicht. Der überwiegende Teil der Fotografen arbeitete in einem Studio und nahm gelegentlich Bilder auf, um sie an die Presse zu verkaufen. ${ }^{59}$ Die Redaktionen erwarben die Publikations-, aber nicht die Bildrechte, wodurch mehrere Zeitschriften die gleiche Fotografie kaufen und abdrucken konnten. Nur wenn ein Lichtbildner von einer Redaktion einen konkreten Auftrag erhielt, trat er auch die Bildrechte ab. ${ }^{60}$ Gerade unter den Portraits, die die Illustrierten abdruckten, sind viele Werke namhafter Fotografen, ${ }^{61}$ und auch Kunstfotografen wie Aleksej Sergeevič Mazurin (1846-unklar) verkauften ihre Aufnahmen an Illustrierte. ${ }^{62}$ Die Redakteure griffen für manche Themen auf Spezialisten wie

$5^{2}$ Beljaev; Zinov'ev; Nikiforov (Hrsg.): Bibliografija periodičeskich izdanij, S. 104.

53 RGIA f. 776 , op. 8, d. 1361, l. 11-16.

54 RGIA f. 776, op. 8, d. 1361, l. 36. Zu Sytins Tätigkeit als Herausgeber siehe auch: Machonina: Istorija russkoj žurnalistiki načala XX veka, S. 113, 115 .

55 Hogarth: The Artist as Reporter, S. 24.

56 Stolarski: The Rise of Photojournalism, S. 56-57.

57 Beispielsweise: o. A.: Bakinskija sobytija. Neftjanyja cisterny, in: Niva, 08.10.1905, S. 795.

58 Beispielsweise: I. P. Voronin: Katastrofa na kostemol'nom zavode v Slobode po Krovskij, Samarskoj gubernii, in: Ogonëk, 16.02.1912, S. 15.

59 Holzer: Rasende Reporter, S. 38.

6o Stolarski: The Rise of Photojournalism, S. 114.

61 Stolarski: The Rise of Photojournalism, S. 99-101.

62 Beispielsweise: A. Mazurin: Dačnica, in: Iskry, 30.o6.19o2, S. 1. 
Karl (August) Andreevič Fišer (1859-1923) zurück, der in Moskau vornehmlich Theaterfotografien anfertigte. ${ }^{63}$

Viele Fotografien von Szenen und Ereignissen aus der russischen Hauptstadt tragen den Vermerk „Bulla“. Die Aufnahmen des ursprünglich deutschen Fotografen Carl Oswald Bulla, in Russland bekannt als Karl Karlovič Bulla (unklar-1929), ${ }^{64}$ und seiner beiden Söhne Viktor (1883-1938) und Aleksandr (1881-1943) waren bei den russischen Illustrierten beliebt. Sie zeichnen sich durch hohe Bildqualität und ästhetisches Gespür aus. Die Familie Bulla baute in den Jahren vor dem Ersten Weltkrieg die erste Fotoagentur im Zarenreich auf. Dabei verfolgten sie eine Doppelstrategie und betrieben einerseits ein Studio, andererseits fertigten sie Fotoreportagen an, die sie der Presse anboten. ${ }^{65} \mathrm{Um}$ die große Nachfrage zu befriedigen und möglichst viele Themengebiete abzudecken, arbeiteten zahlreiche weitere Lichtbildner für das Familienunternehmen und fertigten unter dem Namen Bulla Aufnahmen für die Agentur an. ${ }^{66}$

Bei Berichten über Ereignisse abseits der Metropolen wäre es zu aufwendig gewesen, eigens Fotografen vor Ort zu schicken. Die Herausgeber bedienten sich einer Strategie, die auch im deutschsprachigen Raum verbreitet war: ${ }^{67}$ Sie riefen in Anzeigen lokale Fotografen und Amateure dazu auf, Fotografien aktueller Ereignisse an die jeweilige Redaktion zu senden. ${ }^{68}$ Gerade hier finden sich viele Namen, die nur vereinzelt erscheinen und nicht zu den großen Protagonisten der russischen Fotografieszene zählen. Dies zeigt, dass die Blätter mit dieser Strategie sehr erfolgreich waren.

Oft wird in der Forschungsliteratur behauptet, dass die Fotografie im 19. Jahrhundert als „objektiv“ betrachtet wurde und daraus ihre besondere Faszination geschöpft habe. ${ }^{69}$ Dies mag auf bestimmte Situationen zugetroffen haben, so

63 Šipova: Fotografy Moskvy (2006), S. 236-246.

64 K. Bulla erhielt 1896 den Titel eines „Photographen vom Ministerium des Kaiserhofs“ und bekam außerdem eine Sondererlaubnis, so dass er auch von militärischen Ereignissen Aufnahmen anfertigen konnte. Golovina: Für die Nachkommen aufbewahren, S. 172.

65 Besonders bekannt wurden die Reportagen V. Bullas von der russischen Front während des Russisch-Japanischen Kriegs sowie die Aufnahme K. Bullas von der Oktoberrevolution 1917. Illustration zu: Bol'ševik v Petrograd, in: Iskry, 22.10.1917, S. 326. Stolarski: The Rise of Photojournalism, S. 129-133.

66 Aus diesem Grund ist nicht immer klar, ob beispielsweise die mit F. Bulla ausgezeichneten Abzüge im CGAKFF tatsächlich von einem Mitglied der Familie Bulla stammten oder ob sie ein angestellter Fotograf aufgenommen hatte.

67 Holzer: Rasende Reporter, S. 56.

68 Stolarski: Another Way of Telling the News, S. $568-569$.

69 Beispielsweise: Burke: Augenzeugenschaft, S. 24; Edwards; Hart: Introduction, S. 3; Hartewig: Fotografien, S. 428; Sekula: Reading an Archive, S. 190. 
lobte Leopold von Ranke (1795-1886) Fotografien als die „echtesten unmittelbaren Urkunden “70. Den zeitgenössischen Fotografen war jedoch bewusst, dass ihre Aufnahmen nicht einfach die Realität wiedergaben, sondern dass sie mittels Inszenierung und Bearbeitung versuchten, der Realität möglichst nahe zu kommen. ${ }^{71}$

Gerade in Bezug auf illustrierte Zeitschriften war die Stellung der Fotografie als adäquate Abbildung eines Ereignisses zunächst umstritten. ${ }^{72}$ Die bisher üblichen Zeichnungen und Holzschnitte zeigten oft dramatische Szenen, mit denen die Künstler dem Betrachter das Gefühl gaben, sich mitten in der Szene zu befinden. ${ }^{73}$ Sie vermittelten dem Publikum einen emotionalen Zugang zu einem Ereignis, ohne selbst vor Ort gewesen sein zu müssen. Die fotografische Unmittelbarkeit war anderer Natur. Obwohl die Aufnahmen häufig starr und gestellt wirkten, war die Existenz des Bildes der Beweis für die Anwesenheit des Fotografen vor Ort. ${ }^{74}$ Die emotionale "Objektivität" wurde durch eine technische „Objektivität“ abgelöst. Allmählich gewöhnten sich die Betrachter an die visuellen Besonderheiten der gedruckten Fotografien und das zunächst ungewohnte Bildraster. Am Vorabend des Ersten Weltkriegs galten Fotografien als authentische Abbildungen, obwohl die Technik noch große Mühe hatte, Bewegungen einzufangen.$^{75} 1912$ warb die Zeitschrift Ogonëk damit, dass ihre Bilder das Tagesgeschehen wie in einem Spiegel wiedergäben. ${ }^{76}$

70 Zitiert nach Hartewig: Fotografien, S. 428.

71 Der Fotograf Sergej Aleksandrovič Lobovikov (1870-1941) beschreibt, wie die Menschen ihr Verhalten sofort veränderten, sobald er sich mit der Kamera nähere. Daraufhin entwickelte er eine spezielle Form der Inszenierung, um diesen Effekt auszugleichen. RGIA f. 20, op. 16, d. 191, l. 139. Auch Pohlmann beobachtet, dass in den Niederlanden Fotografen sich bewusst waren, dass Fotografien keine objektiven Bilder darstellten. Pohlmann: Industriebilder, S. 11.

72 Dies galt auch für den wissenschaftlichen Kontext, in dem sich die Fotografie erst langsam als bevorzugtes Medium der Dokumentation und Beweisführung durchsetzte. Lorraine Daston; Peter Galison: Objektivität, Frankfurt a. M. 2007, S. 121-132.

73 Holzer: Rasende Reporter, S. 55; Wolfgang Pensold: Eine Geschichte des Fotojournalismus. Was zählt, sind die Bilder, Wiesbaden 2015, S. 7-8. Bourdieu betont, dass sich die Menschen Ende des 19. Jahrhunderts darauf geeinigt hätten, dass Fotografien ein Modell der Wirklichkeit abbildeten und objektiv seien. Peter Geimer: Theorien der Fotografie. Zur Einführung, Hamburg ${ }^{2} 2010$, S. 75 .

74 Holzer: Rasende Reporter, S. 55.

75 Beegan: The Mass Image, S. 187, 196.

$76 \quad$ o. A.: O podpiske na 1913 g., in: Ogonëk, o6.01.1913, Anhang. 


\subsection{Keine Verkaufsschlager - Fabrik, ein seltenes Motiv}

Für die Herausgeber der Illustrierten war es entscheidend, den Geschmack ihrer Leser zu treffen. Gefiel diesen der Inhalt nicht, wechselten sie zur Konkurrenz. ${ }^{77}$ Viele Illustrierte hatten nur eine kurze Lebensdauer und selbst die beliebte Zeitschrift Niva kam in den Jahren vor dem Ersten Weltkrieg in finanzielle Schwierigkeiten, weil sie zunehmend Leser verlor. ${ }^{78}$ Entsprechend ihrem Anspruch, die Leser zu unterhalten, legten die Redakteure besonderen Wert auf eine Themenwahl, die sich an der Freizeitbeschäftigung der städtischen Mittelschicht orientierte: Theater, Bildende Kunst, Sport und Literatur. Dem Motiv der Fabrik wurde nur wenig Interesse entgegengebracht. ${ }^{79}$ Andere Aspekte der Industrialisierung waren jedoch in der Berichterstattung zwischen 1900 und 1914 präsent. Beispielsweise informierten die Journalisten ihre Leser über neue technische Entwicklungen bei der Eisenbahn, der Telegrafie oder in der Luftfahrt. ${ }^{80}$

Bereits in der Zeit der Holzschnitte zählten Fabrikdarstellungen nicht zu den Kernthemen der Berichterstattung. Als Vergleichsgegenstand dient die Zeitschrift Vsemirnaja Illjustracija (Die weltweite Illustrierte), ${ }^{81}$ deren Ausgaben von 1870 bis 1880 für das vorliegende Kapitel ausgewertet und als Vergleich herangezogen wurden. Die Analyse zeigt, dass in der vorfotografischen Periode der Illustrierten Fabriken mit 15 Artikeln und Bildstrecken vertreten, aber kein zentrales Thema waren. Einerseits ließe sich das geringe Interesse damit erklären, dass die Industrie in Russland in den $1870 e r$ Jahren noch deutlich weniger präsent war als um 1900. Andererseits hätte es auch in dieser Periode bereits lohnende Motive gegeben, beispielsweise den Besuch des Zaren Alexanders II. in der Tulaer Waffenfabrik. Offenbar war das Interesse an den Leistungen der russischen Fabriken im Zarenreich gering. Damit unterschieden sich russische Publikationen von Zeitschriften, die zur selben Zeit in Deutschland erschienen. Die konservative Illustrierte Zeitung Leipzig

77 Daniel Brower: The Penny Press and it's Readers, in: Stephen Frank; Mark Steinberg (Hrsg.): Cultures in Flux. Lower-Class Values, Practices, and Resistance in Late Imperial Russia, Princeton 1994, S. 146-167, S. 150.

78 Stolarski: The Rise of Photojournalism, S. 55-56, 235.

79 Diese Beobachtung gilt auch für Fotoreportagen in französischen Zeitschriften. Gunthert: La photographie, ou l'accès de l'industrie au pittoresque, S. 44.

8 Z Zum Beispiel: Iskry, 25.02.1901, S. 116-117; Novoe Vremja, 06.06.1909, S. 10.

$81 \quad 1869$ erschien die konservative Illustrierte erstmals. Die Wochenzeitschrift stellte 1898 ihr Erscheinen ein, weil sie mit den kostengünstigeren Illustrierten nicht konkurrieren konnte. L. K. Švecova: Massovye eženedel'niki dlja pestrago čitatelja, in: B. A. Bjalik (Hrsg.): Literaturnyj process i russkaja žurnalistika konca XIX-načala XX veka, 1890-1904, Moskva 1982, S. 275-297, S. 278-279. 
informierte ihre Leser von den $1840 e r$ bis in die 189oer Jahre über technische Neuerungen. ${ }^{82}$ Die populäre Illustrierte Gartenlaube berichtete von den $1860 e r$ bis in die $1890 e r$ Jahre regelmäßig über die deutsche Industrie. ${ }^{83}$ In beiden Blättern war der Grundtenor der Beiträge überwiegend positiv. ${ }^{84}$ Auch in der französischen Presse bildeten Industriedarstellungen einen ähnlich festen Bestandteil in der Berichterstattung. ${ }^{85}$ Von den späten 185 oer bis in die $1870 \mathrm{er}$ Jahre gaben Zeitschriftenherausgeber aufwendig illustrierte Publikationen heraus, die französische Musterbetriebe vorstellten. ${ }^{86}$

1871 begann Vsemirnaja Illjustracija eine Reihe von Holzstichen zu veröffentlichen, die unter dem Titel "Unsere Fabriken und Handwerksbetriebe“ standen. Allerdings schien diese Reihe nicht langfristig erfolgreich zu sein. Nach sechs Beiträgen im ersten Jahr erschien nur noch eine Illustration im Februar $1873^{87}$ und eine im Mai $1876 .{ }^{88}$ Es stellte sich keine Kontinuität ähnlicher Darstellungen ein. Wie oben ausgeführt, könnte ein Deutungsangebot sein, dass Industriebetriebe im Bewusstsein der Leserschaft noch zu wenig präsent waren, um eine solche Serie zu rechtfertigen. Denkbar wäre auch, dass die Illustrationen in der eher industriekritischen und konservativen Leserschaft, auf die das Blatt abzielte, auf wenig Begeisterung stießen oder dass Fabriken nicht Teil des russischen Selbstverständnisses waren.

82 Franz Dröge; Andreas Wilkens: Populärer Fortschritt. 150 Jahre Technikerberichterstattung in deutschen illustrierten Zeitschriften, Münster 1991, S. 37.

83 Kirsten Belgum: Popularizing the Nation. Audience, Representation, and the Production of Identity in Die Gartenlaube, 1853-1900, Lincoln, London 1998, S. 107, 114-115.

84 Zwar differenzieren die Autoren nicht zwischen unterschiedlichen Facetten der Industrialisierung (eine genaue Bewertung von Fabriken lässt sich aus der Studie nicht rekonstruieren), negative Bewertungen werden aber insgesamt bis 1913 so gut wie nie geäußert. Dröge; Wilkens: Populärer Fortschritt, S. 54, 73-74.

85 In Frankreich wurden Zeitungen und Zeitschriften in einen engen Bezug zur Industrialisierung gesetzt, sie galten als Produkte der Moderne. Vincent Duclert: L'homme de science et le débat public au XIX ${ }^{\text {e }}$ siècle, in: Dominique Kalifa u. a. (Hrsg.): La civilisation du journal. Histoire culturelle et littéraire de la presse française au XIX ${ }^{\mathrm{e}}$ siècle, Paris 2011, S. 1491-1498, S. 1491.

86 Ivan Grassias: Les Grandes Usines de Turgan. Un auteur, un corpus, des images, in: Denis Woronoff (Hrsg.): Les images de l'industrie de 1850 à nos jours. Actes du colloque tenu à Bercy, les 28 et 29 juin 2001, Paris 2002, S. 32-41, S. 33-34. Zur Begeisterung für Industrie in der französischen Gesellschaft: Vogel: Mythos Moderne, S. 110-112.

87 A. K. Beggrok; K. Beeržak: Naši zavody i masterskija. 3.ooo pudivoj parovok molot Obuchovskago stalilitejnago zavoda, v S.-Peterburge, in: Vsemirnaja Illjustracija, 17.02.1873, S. 124 .

88 Chr. Talja; K. I. Tichomirov; Ė. Dammjuller: Naši zavody i masterskija. Suchogorskij zavod I. G. A. Pastuchova na Urale. Obščij vid zavoda, in: Vsemirnaja Illjustracija, 15.05.1876, S. 385 . 
Von den sechs analysierten Zeitschriften veröffentlichte nach der Jahrhundertwende keine vergleichbare Darstellungen und Berichte wie deutsche oder französische Blätter. Einen ähnlich positiven Unterton wie ihre westeuropäischen Kollegen stimmten russische Journalisten nur in den Berichten über die Firmenjubiläen des Tul'skij Imperatora Petra Velikago Oružejnyj zavod oder des Baltijskij zavod an. Darüber hinaus berichteten die Journalisten über zwei Jubiläen deutscher Firmen: Borsig und Krupp. Beide Betriebe unterhielten wichtige Kontakte zur russischen Industrie, Borsig besaß sogar eine eigene Produktionsstätte im Zarenreich. ${ }^{89}$ Krupp war ein weltweit agierendes Unternehmen, und diese internationale Bedeutung machte das Firmenjubiläum 1912 zu einem gesellschaftlichen Ereignis über Deutschland hinaus. Positiv berichteten die Illustrierten auch über Unternehmen, die mit der Zeitungsproduktion in Verbindung standen - beispielsweise Fabriken von Druckplatten für Kunstdrucke. ${ }^{90}$

Die wenigen Artikel, die ohne besonderen Anlass über Fabriken berichteten, widmeten sich der Nahrungsmittel- oder Konsumgüterindustrie überwiegend aus den Regionen Moskau und St. Petersburg. Die Herausgeber konnten damit auf ein lokales Interesse des Publikums bauen. ${ }^{91} \mathrm{Im}$ Juli 1914 berichtete Ogonëk über den Imperatorskij farforovyj i stekljanyj zavod (Kaiserliches Porzellan- und Glaswerk). ${ }^{92}$ Die Fotografien aus der Porzellanproduktion, die Arbeiter beim Formen und Bemalen der zerbrechlichen Gefäße zeigen, erinnern stärker an ein Künstleratelier als an eine Fabrik. Hinweise auf moderne Industriearchitektur oder Verwendung von Maschinen im Herstellungsprozess fehlen vollständig. Stattdessen zeigen eineinhalb Seiten des vierseitigen Artikels Fotografien von Produkten oder Ausstellungräumen. Obwohl der Artikel über eine Fabrik berichtet, lässt die verwendete Bildsprache Charakteristika der Fabrikfotografie vermissen und orientiert sich an der visuellen Darstellung Bildender Kunst.

Werften, die Fertigung von Schiffen ${ }^{93}$ oder die Herstellung von Heißluftballons waren weitere Themen mit Industriebezug. ${ }^{94}$ Die große Anzahl dieser Artikel legt nahe, dass Reportagen und Meldungen über Themen der Marine und Aviatik bei russischen Lesern beliebt waren, wobei letztere eine besondere

89 Zur Geschichte der Firma Borsig: Kurt Pierson: Borsig, ein Name geht um die Welt. Die Geschichte des Hauses Borsig und seiner Lokomotiven, Berlin 1973.

$90 \quad$ o. A.: Fabrika Č. Gofmana v Peterburge, in: Niva, 23.6.1907, S. 418.

91 Bösch: Mediengeschichte, S. 112.

92 Mit Fotografien von I. Ocup: Na Imperatorskom farforovom i stekljanom zavode, in: Ogonëk, 13.07.1914, S. 4-7. Seit seiner Gründung 1744 produzierte das Unternehmen in St. Petersburg Porzellan und Glaswaren.

93 o. A.: K spusku drednouta "Sevastopol'“, in: Novoe Vremja, 18.o6.1911, S. 8.

94 o. A.: Kak delajut' vozdušnyj šar, in: Niva, 27.12.1908, S. 919. 
Faszination auf sie ausübte. ${ }^{95}$ Die Begeisterung für die Luftfahrt ist kein spezifisch russisches Phänomen, sie findet sich zeitgleich im Habsburgerreich und in Deutschland. ${ }^{96}$ Die erfolgreichen Flugversuche im 19. Jahrhundert rückten die Erfüllung des Traums vom Fliegen plötzlich in greifbare Nähe. ${ }^{97}$ Für die russischen Leser symbolisierte die Luftfahrt sowohl das Aufbrechen in unbekannte Gefilde, das Bezwingen der Natur, als auch eine wichtige technische Entwicklung, die dafür sorgen sollte, dass das Zarenreich in die Reihe der modernen westeuropäischen Staaten aufrückte. ${ }^{98}$ Außerdem betonte die Presse die Bedeutung dieser Technik für das Militär und die Kriegsführung. ${ }^{99}$ In diesen Fällen profitierte das Motiv der Fabrik von der Beliebtheit anderer Themen, hier Marine und Aviatik, und trug in diesem Kontext zur Unterhaltung des Publikums bei.

\section{7·3 Die brennende Fabrik als Sensation}

Berichte über Unfälle gehörten zum festen Bestandteil der russischen Illustrierten. Sie folgten damit einem Trend, der europaweit in der Massenpresse vorherrschte: die Gier nach Sensationsmeldungen. ${ }^{100}$ Dies war nichts prinzipiell Neues, im 19. Jahrhundert hatten Fotografen frühzeitig bemerkt, dass Aufnahmen von Verkehrsunfällen oder Naturkatastrophen viele Käufer fanden. ${ }^{101}$ Mit dem Aufkommen der illustrierten Presse und der günstigen Massenpresse erhielt die Sensationsgier jedoch eine neue Qualität. Die Medien kannten die Beliebtheit entsprechender Meldungen, und konnten mit diesen

95 Ingold: Der große Bruch, S. 56-64; Jurij Leving: Vokzal, Garaž, Anger. Vladimir Nabokov i poètika russkogo urbanizma, Sankt-Peterburg 2004, S. 293.

96 Holzer: Rasende Reporter, S. 91-104; Sabine Höhler: Luftfahrtforschung und Luftfahrtmythos. Wissenschaftliche Ballonfahrt in Deutschland 1880-1910, Frankfurt a. M. 2001, S. 59 .

97 Beim Traum vom Fliegen handelt es sich um einen Mythos, der die Menschen schon lange vor dem 19. Jahrhundert faszinierte. Wolfgang Behringer; Constance Ott-Koptschalijski: Der Traum vom Fliegen. Zwischen Mythos und Technik, Frankfurt a. M. 1991, S. 21; Höhler: Luftfahrtforschung und Luftfahrtmythos, S. 58 .

98 Palmer: Dictatorship of the Air, S. 10, 20-21, 55 .

99 Von Hardesty: Early Flight in Russia, in: Robin Higham; John Greenwood; ders. (Hrsg.): Russian Aviation and Air Power in the Twentieth Century, London 1998, S. 18-36, S. 24.

100 Holzer: Rasende Reporter, S. 29; Lenger: Metropolen der Moderne, S. 228; Kasper: Belichtung und Wahrheit, S. 13-14.

101 Uta Grefe führt dies auf die zunehmende Kommerzialisierung der Fotografie zurück. Uta Grefe: Die Geschichte der Architekturfotografie des 19. Jahrhunderts. Architekturfotografie - Architekturmalerei, Köln, Univ., Diss., 1980, S. 115-116, Starl: Im Prisma des Fortschritts, S. 105. 
Themen ihre Auflagenzahl erhöhen. ${ }^{102}$ Russische Journalisten verfassten ihre Berichte oft in einem eher neutralen Sprachstil, dennoch beweist die Häufigkeit solcher Artikel, dass sie den Sensationalismus des Publikums befriedigen sollten. ${ }^{103}$ Auch in den Aufrufen für Bildbeiträge, die russische Zeitschriften an ihre Leser schickten, interessierten sich die Redaktionen besonders für Fotografien von Katastrophen. ${ }^{104}$

Die Fotografien sollten möglichst spektakulär sein, wie beispielsweise Bilder von brennenden Ölquellen. ${ }^{105}$ Am beliebtesten waren Berichte über Eisenbahnunglücke. Die Eisenbahn war eines der zentralen Symbole der fortschreitenden Modernisierung des Zarenreichs. ${ }^{106}$ Fotografien von Eisenbahnunglücken illustrierten erstmals die selbstzerstörerische Kraft von Maschinen.107 Aufnahmen der ineinander verkeilten oder umgestürzten Wagen erschienen so häufig in der Presse, dass Karikaturisten sich sogar darüber lustig machten. ${ }^{108}$

Ausgehend von Eisenbahnunglücken entwickelte sich eine Bildsprache, die als Maßstab für die Fotografie von Unglücken angesehen werden kann. Zugunglücke boten interessante ästhetische Elemente: Umgestürzte und verkeilte Waggons und Lokomotiven bilden starke, ungeordnete Linien, die die Aufnahmen dominieren (Abb. 50 und 51).

Diese chaotische Linienführung erhöht den Eindruck von den Ausmaßen der Zerstörung. Häufig ist sogar die Unterseite der Wagen und Lokomotiven sichtbar. In diesen Fällen ist in den Bildern die bekannte Ordnung vollständig aufgehoben und das Unterste zuoberst gekehrt. Dies wirkt besonders eindrücklich, weil die aus Metall- und Holzteilen gefertigten Zugwaggons zwar durch den Unfall aus ihrer bekannten horizontalen Ordnung gerissen, aber nicht durch Feuer entstellt sind. Die Zeitgenossen konnten problemlos nachvollziehen, wie groß der Schaden war, weil sie das Aussehen eines funktionsfähigen Zuges kannten. Neben den verunglückten Zügen zeigt die Mehrheit dieser Fotografien auch Menschen, die vor Ort waren, als der Fotograf den Auslöser betätigte. In dem Durcheinander von Formen sind diese Personen

102 Bösch: Mediengeschichte, S. 116.

103 Marc Elie; Klaus Gestwa: Zwischen Risikogesellschaft und Katastrophenkulturen. Zur Einführung in die Katastrophengeschichte des östlichen Europas, in: Jahrbücher für Geschichte Osteuropas, Jg. 62/2014, Heft 2, S. 161-179, S. 162; Stolarski: Another Way of Telling the News, S. 568; Steinberg: Petersburg, S. 35 .

104 Stolarski: Another Way of Telling the News, S. 568-569.

105 Beispieslweise: Niva, 08.10.1905, S. 792.

106 Hierzu beispielsweise: Cvetkovski: Modernisierung durch Beschleunigung; Schenk: Russlands Fahrt in die Moderne; Sperling: Der Aufbruch der Provinz.

107 Michael Freeman: Railway and the Victorian Imagination, New Haven, London 1999, S. 84; Heßler: Kulturgeschichte der Technik, S. 178.

108 Siehe: o. A.: Na železnych dorogach pri stolknovenii poezdov, in: Budil'nik, 27.08.1900, S 2. 


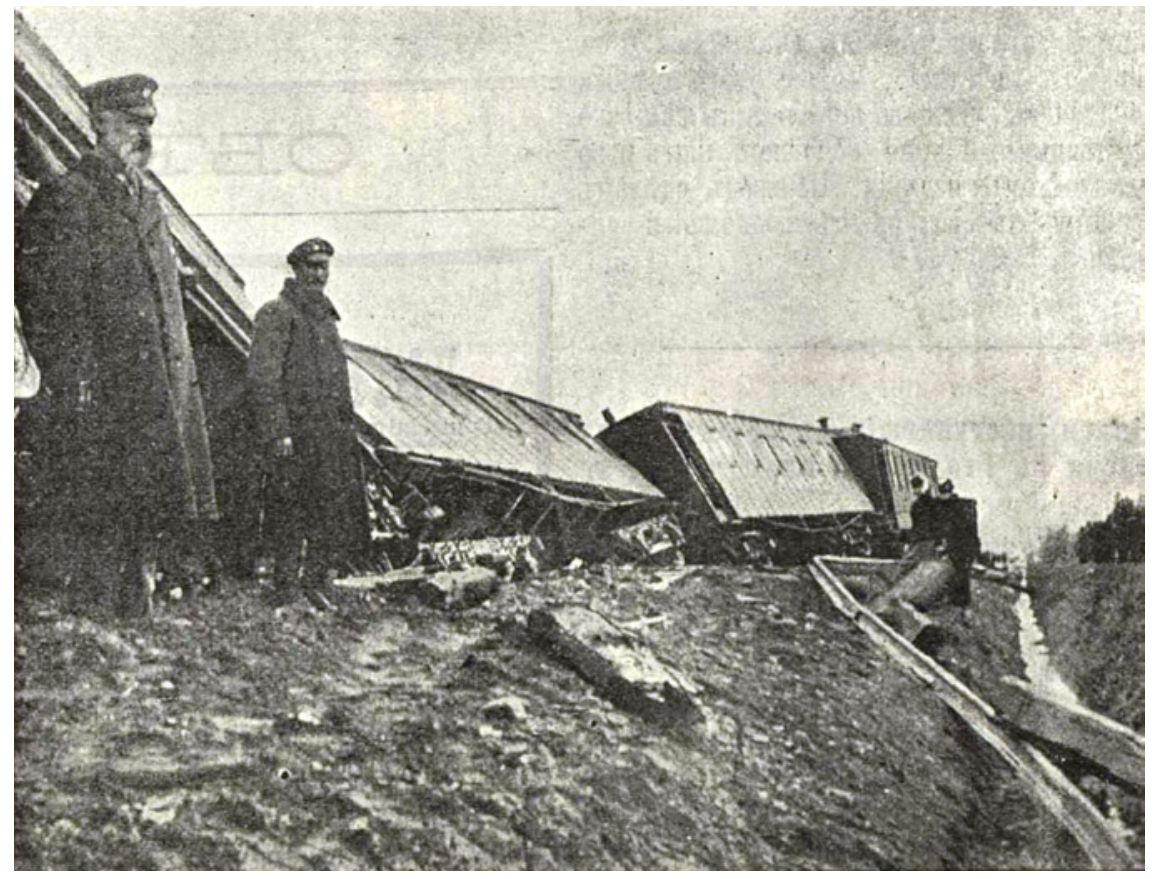

Abb. $5^{0} \quad$ o. A.: Entgleisung eines Zugs der Baltischen Eisenbahn. 2. April. Wagons nach der Entgleisung des Zugs (Krušenie poezda Baltijskoj žel. dor. 2-go aprelja. Vagony poterpevšago krušenie poezda), in: Novoe Vremja, 11.04.1901, S. 11

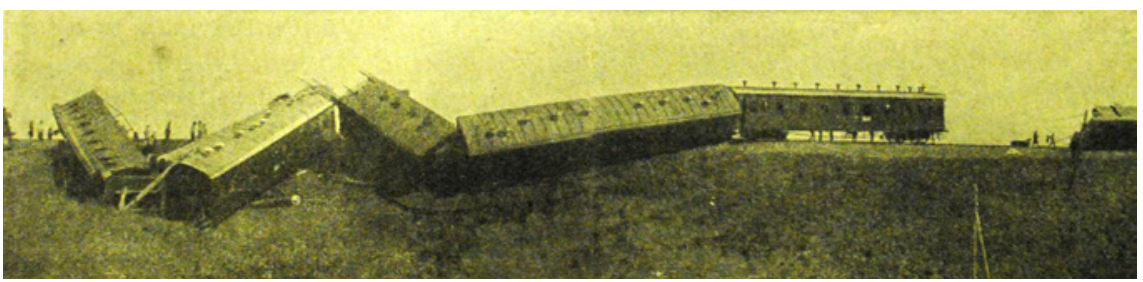

Abb. $5^{1} \quad$ Kamennovyj: Nach der Katastrophe. Ein in der Mitte in zwei Teile gebrochener Wagen der 1. und 2. Klasse. Rechter Hand ein noch auf den Geleisen stehender Wagon (Posle katastrofy. Posredine perelomivšijsja nadvoe vagon-mikst I-II kl. Sprava videnustojavšij na rel'sach vagon), in: Ogonëk, 18.06.1911, S. 13

teilweise die einzigen vertikalen Elemente, die parallel zur Bildkante stehen (Abb.50). Sie bilden den Kontrapunkt zum Chaos des Bildinhalts und symbolisierten das Überleben einer Ordnung in der Katastrophe.

Feuer war ein beliebtes Sujet in russischen Zeitungen.109 Für Brände in Fabriken entwickelte sich im Gegensatz zu Eisenbahnunfällen keine

109 Steinberg: Petersburg, S. 73. 
einheitliche Bildsprache. Es schien für die Lichtbildner schwierig gewesen zu sein, deutlich zu machen, wodurch sich die neuen Dimensionen der Zerstörung im Vergleich zu bisherigen Brandkatastrophen auszeichneten. ${ }^{110}$ Für die Ausbildung einer einheitlichen visuellen Darstellung kam erschwerend hinzu, dass Industriebauten sehr unterschiedlich waren und Brände die architektonischen Details verwüsteten. Dem Publikum erschloss sich das Ausmaß der Verwüstung darum nur, wenn die Betrachter entweder die Fabrik kannten oder die Redakteure eine Fotografie des betroffenen Gebäudes vor dem Brand beifügten. ${ }^{111}$ Teilweise verzichteten die Zeitschriften ganz auf eine Darstellung des Feuers und druckten lediglich eine Ansicht der betroffenen Gebäude vor der Zerstörung. ${ }^{112}$ Möglicherweise hatten die Fotografen keinen Zugang zum Fabrikgelände und zum Unglücksort, oder die Ruinen waren so wenig aussagekräftig, dass die Redaktion von einer Publikation absah. Hohe Schadenssummen oder die Zahl der Opfer der Katastrophe erwähnten die Autoren meist nur in den Begleittexten oder Bildunterschriften. ${ }^{113}$ Die Aufnahmen zeigen häufig schaulustige Menschen (Abb. 52), die vor noch brennenden oder ausgebrannten Gebäude stehen.

Wie auf Abbildung $5^{2}$ sind häufig im Hintergrund noch senkrechte architektonische Elemente zu erkennen, und die Gebäudekanten sowie die senkrechten Elemente der Fenstergewände greifen die Senkrechte der Bildkanten auf. Dadurch stechen die Personen weniger ins Auge, als dies bei Zugunglücken der Fall ist.

Bei Abbildung 53 verdeutlichte der Fotograf die Ausmaße des Brands in seiner Aufnahme für die Zeitschrift Iskry, indem er große Schuttberge in den Vordergrund rückte. ${ }^{114}$ Im schwarzen Vordergrund sind nur schemenhaft Konstruktionselemente wie Balken zu erkennen. Dagegen hebt sich im Hintergrund eine ausgebrannte Fassade ab, die vormals zu einer Werkshalle gehörte. Die Fabrikfassade erscheint als Gerippe mit leeren Fensteröffnungen, jeglicher

$110 \mathrm{Zu}$ Fotografien von Fabrikunglücken siehe auch: Micheline Nilsen: Architecture in Nineteenth-Century Photographs. Essays on Reading a Collection, Farnham, Burlington 2011, S. 84-85.

111 Beispielsweise: o. A.: Požar na ižorskoj pisčebumažnij fabrike, in: Niva, 05.03.1903, S. 517.

112 Beispielsweise: o. A.: K požaru na Obuchovskom stalelitejnom zavode, in: Novoe Vremja, 13.04.1908, S. 7 .

113 Beispielsweise: o. A.: Požar Chamovničeskago pivovarennago zavoda v Moskve, in: Iskry, 09.03.1908, S. 78 .

114 A. I. Sosel'es: Požar na Mytiščenskom zavode, in: Iskry, 07.08.1911, S. 238. Die Inszenierung der Fotografien erinnert an Darstellungen nach Erdbeben, beispielsweise an Zeichnungen nach dem Erdbeben von Lissabon 1755. Dort hatten auch Feuer die Innenstadt verwüstet. Jan Kozák; Vladimír Čermák: The Illustrated History of Natural Disasters, Dordrecht 2010, S. 134, 141 . 


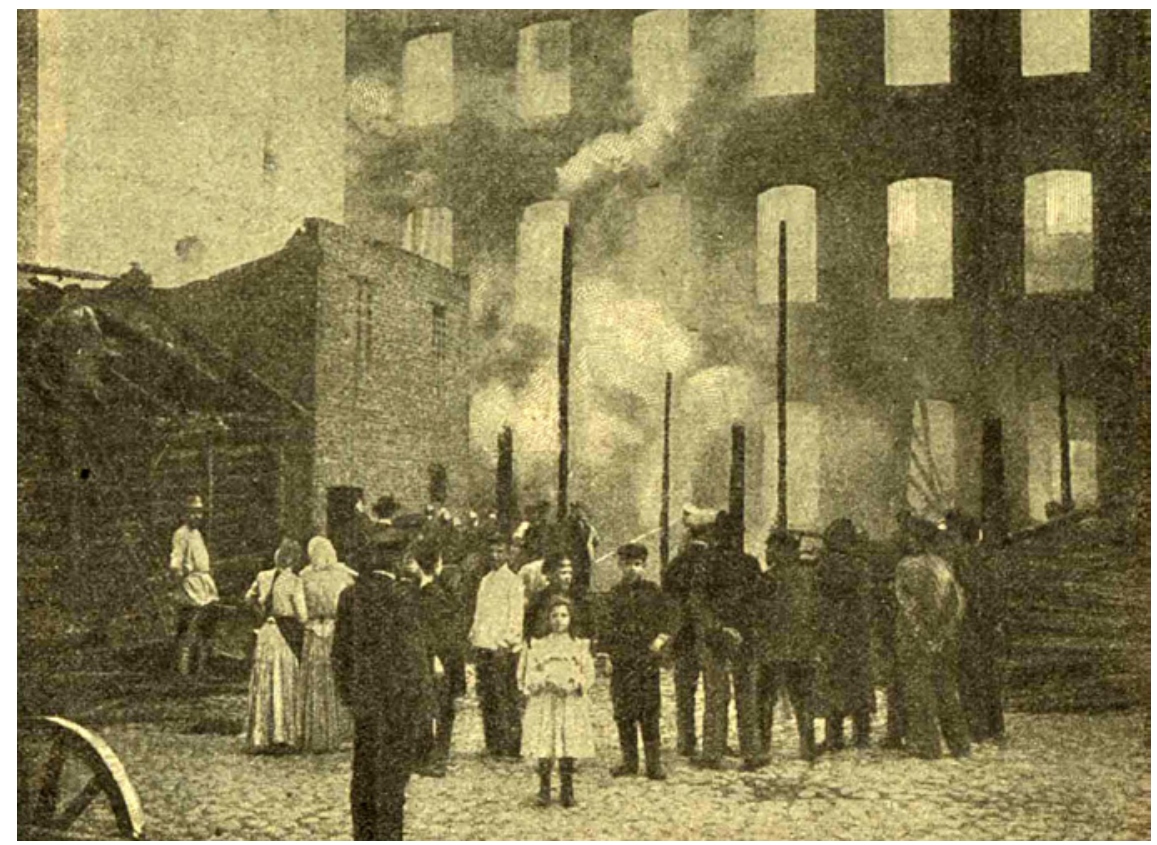

Abb. $5^{2}$ o. A.: Brand im Sägewerk des Nachfolgers Beljaevs (Požar na lesopil'nom zavode naslednikov Beljaeva), in: Novoe Vremja, 25.06.1905, S. 11

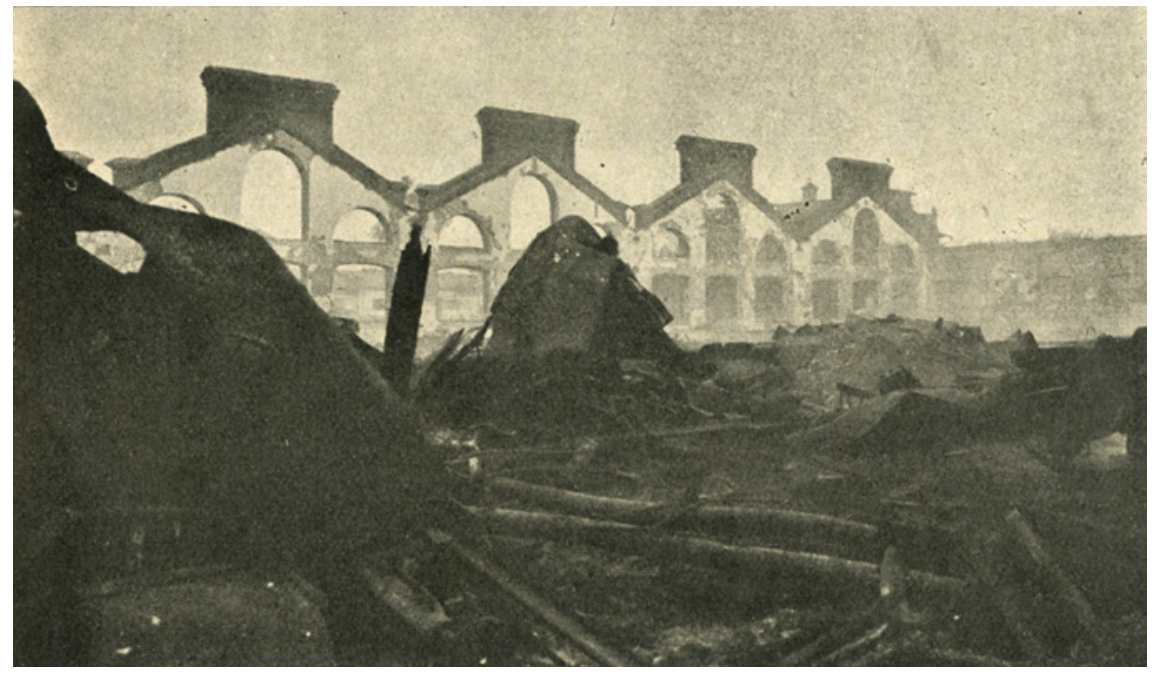

Abb. 53 A. I. Savel'ev: Brand im Mytiščenskij Betrieb. Montageabteilung (Požar na Mytiščenskom zavode. Sbornyja masterskija), in: Iskry, 07.08.1911, S. 238 
klar zuordenbaren Funktion enthoben. Solche durch Unglücke entstandene Mondlandschaften beeindruckten Redakteure und Fotografen.

Die Fotografien von Eisenbahnunfällen und Fabrikbränden spiegeln die Faszination für die zerstörerischen Kräfte, die bei den Unglücken am Werk waren. Verbogene Stahlelemente entwickelten sich zu einem zentralen Element dieser Katastrophenfotografie. Bei der Eisenbahn waren es oft Schienen, die aus ihrer Verankerung gerissen und zu bizarren Formen gebogen waren. ${ }^{115}$ Auf Aufnahmen ausgebrannter Fabriken ragten Eisenträger oder Maschinenteile in merkwürdigen Anordnungen in die Höhe. Im rechten Bild von Abbildung 54 skizzieren einige noch erhaltene Stahlträger das vormalige Aussehen der Halle.

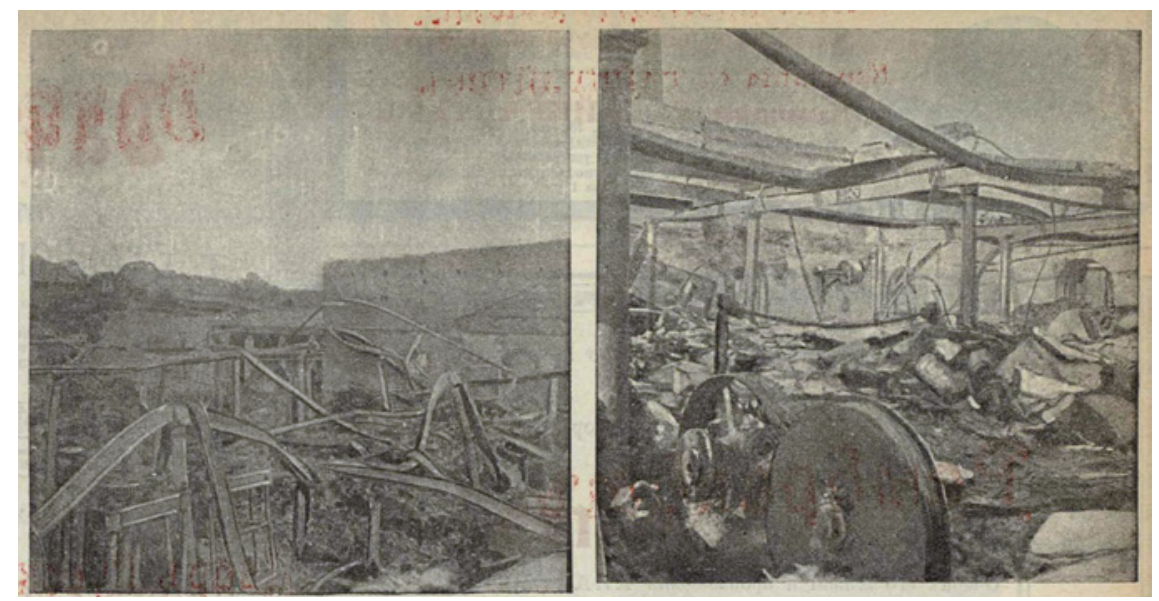

Abb. 54 o. A.: Ansicht der Fabrik der Gesellschaft der Ižorsker Papierfabrik K. i. Ch. Nebe am Fluss Ižor nach dem Brand. Sortierabteilung, Mischkammer (Vid fabriki t-va Ižorskoj pisčebumažnoj fabriki K. i. Ch. Nebe na reke Ižore posle požara. Sortirovočnoe otdelenie, Masso-podgotovitel'noe otdelenie), in: Novoe Vremja, 14.06.1903, S. 11

Im linken Bild sind allein die verschlungenen Stahlelemente übrig geblieben. Der durch die enorme Hitze des Feuers aus der Form geratene Stahl, der mit extremer Härte und Haltbarkeit assoziiert wurde, erscheint hier formbar wie Wachs. In diesem Kontext des Sensationismus nahmen Fabriken einen wichtigen Platz innerhalb russischer Illustrierter ein.

115 Sehr ähnliche Inszenierungen erschienen in Illustrierten Zeitschriften nach dem Erdbeben und dem anschließenden Brand in San Francisco 1906. Kozák; Čermák: The Illustrated History of Natural Disasters, S. 178-179. 
Die Berichterstattung über Fabrikunglücke führte in den russischen Illustrierten kaum zu Kritik an der kapitalistischen Wirtschaftsweise. ${ }^{116}$ Insgesamt waren Redakteure mit Kritik an der Industrialisierung insbesondere bis $1906 \mathrm{sehr}$ zurückhaltend. In dieser Beziehung unterschieden sich die russischen Zeitschriften von vergleichbaren Publikationen beispielsweise in den USA. Dort waren Pressefotografien sehr stark mit Sozialkritik verbunden. ${ }^{117}$ Es mangelte im Zarenreich nicht an Motiven: Streiks, Darstellungen der schlechten Lebensund Arbeitsbedingungen der Menschen, Umweltverschmutzung oder die Verbreitung von Krankheiten hätten sich als Ansatzpunkte für eine kritische Auseinandersetzung mit Missständen angeboten. Statt dieser Themen kann als Zeichen der Kritik allenfalls die Tatsache interpretiert werden, dass es kaum Artikel gab, die ausdrücklich positiv über Industrie berichteten.

Bereits in der vorfotografischen Zeit gestanden die Redakteure der Vsemirnaja Illjustracija den Schattenseiten der Industrialisierung wenig Raum zu. Auch über die Situation der städtischen Unterschicht Russlands berichtete das Blatt nicht. Die Vsemirnaja Illjustracija zeigte 1872 ihren Lesern die Zustände in einem Berliner Vorort (Abb. 55). ${ }^{118}$

Der Stich verdeutlicht beispielhaft, welche Vorteile die ältere Bildtechnik gegenüber der Fotografie hatte: Der Künstler konnte in einem einzigen Bild mehrere Ebenen des Lebens der Unterschicht darstellen. $\mathrm{Zu}$ sehen sind aus groben Brettern notdürftig gebaute Hütten, auf der Straße kochende Frauen sowie auf der Erde spielende Kleinkinder. Im Hintergrund sitzt eine Gruppe um einen Tisch, auf dem eine Flasche steht, die auf Alkoholkonsum hindeutet. ${ }^{119}$ Ein Fotograf hätte aufgrund mangelnder Tiefenschärfe mehrere

116 Eine Ausnahme bildet der Artikel über ein Fabrikunglück in einer Knochenmühle der Stadt Pokrovsk, seit 1931 Engels. Dort war am 02.06.1912 eine Wand eingestürzt und hatte fast 40 Menschen unter sich begraben. Einige Tage vor dem Ereignis hatten Arbeiterinnen die Fabrikleitung auf Risse im Mauerwerk aufmerksam gemacht. Die Administration ignorierte die Meldung jedoch. Es ist der einzige Fall, in dem ein Autor direkt die Fabrikleitung für einen Unfall verantwortlich machte. Foto I. P. Voronina: Katastrofa na kostemol'nom zavode v slobode Pokrovskoj, Samarskoj gubernii, in: Ogonëk, 16.o6.1912, S. 15 .

117 Bösch: Mediengeschichte, S. 118.

118 A. Daugel' (Stich): Berlin - Ulica barakov v predmet'i goroda, in: Vsemirnaja Illjustracija, og.09.1872, S. 176 .

119 Zum Alkoholismus im Zarenreich bis zur Bauernbefreiung: David Christian: Living Water. Vodka and Russian Society on the Eve of Emancipation, Oxford 1990; zur Bedeutung, die Arbeiter dem Alkoholkonsum zuschrieben: Steinberg: Proletarian Imagination, S. 87-89; zum Umgang mit Alkoholismus in der russischen Literatur: Mark Lawrence Schrad: 


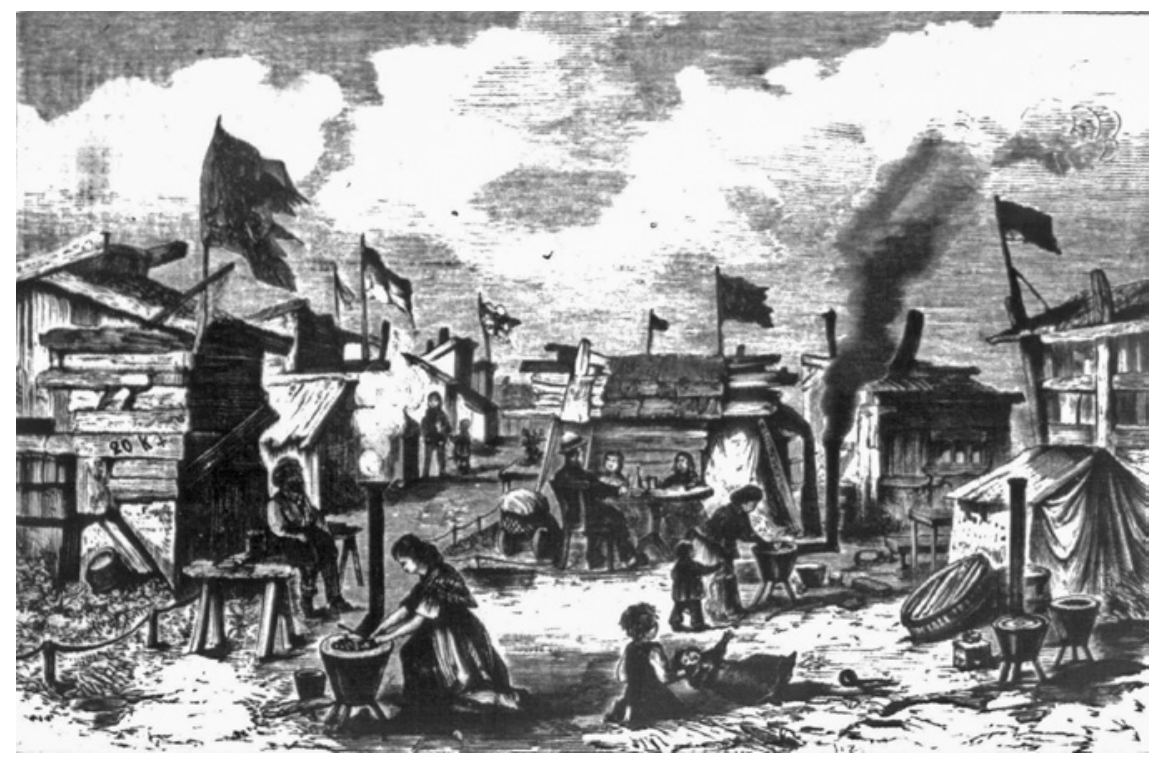

Abb. 55 A. Daugel: Berlin. Eine Straße mit Holzhütten in einem Vorort der Stadt (Berlin. Ulica barakov v predmest'i goroda), in: Vsemirnaja Illjustracija, 09.09.1872, S. 176

Bilder anfertigen und diese in einer Fotomontage kombinieren müssen, um die unterschiedlichen Themen und Ebenen darzustellen. Der Stich schildert erstaunlich schonungslos die Situation der deutschen Unterschicht. Die Darstellung der Misere der Armen konnte in einer russischen Illustrierten wohl nur deshalb erscheinen, weil es sich nicht um Verhältnisse im Zarenreich handelte.

Die Durchsicht aller Fotografien und Texte bestätigt, dass abgesehen von Fabrikunglücken bis 1906 nur ein einziger Bericht zu sozialen Missständen erschien. ${ }^{120}$ Die Illustrationen zeigen Szenen aus dem nächtlichen Leben von Obdachlosen in London und thematisieren somit Probleme im Ausland. Obwohl auch im Zarenreich in den urbanen Zentren viel zu wenig Wohnraum zur Verfügung stand, ${ }^{121}$ erwähnt die Bildunterschrift dies mit keinem Wort. Der Bericht aus der britischen Hauptstadt betraf nicht das direkte Umfeld der Leserschaft, galt folglich als unkritisch und konnte erscheinen.

Vodka Politics. Alcohol, Autocracy, and the Secret History of the Russian State, Oxford 2014, S. 127-140. Zu Antialkoholkampagnen: Patricia Herlihy: The Alcoholic Empire. Vodka and Politics in Late Imperial Russia, Oxford 2002, S. 111-128.

120 o. A.: London nočju, in: Novoe Vremja, 23.10.1902, S. 8-9.

121 Goehrke: Auf dem Weg in die Moderne, S. 296-299. 
Als Folge der Revolution hob die russische Regierung 1906 die Vorzensur auf. ${ }^{122}$ Noch im selben Jahr erschienen deutlich mehr Beiträge in russischen Illustrierten, in denen die Autoren und Fotografen ihr Publikum über gesellschaftliche Probleme informierten. Im ganzen Zarenreich erschienen mehr kritische Publikationen als jemals zuvor. ${ }^{123}$ In Illustrierten blieb es jedoch weiterhin die Ausnahme, direkt Verantwortliche für Missstände zu benennen.

Am deutlichsten äußerten Karikaturisten ihre Kritik an der Industrialisierung. ${ }^{124}$ Die Karikaturenzeitschrift Budil'nik (der Wecker) griff sowohl die Themen Arbeitskampf und Streik, ${ }^{125}$ Nachtasyle ${ }^{126}$ als auch Umweltverschmutzung auf. ${ }^{127}$ In der illustrierten Beilage der Novoe Vremja setzten sich im Untersuchungszeitraum drei Zeichner mit den schlechten Lebensbedingungen in unmittelbarer Nähe von Industrieanlagen auseinander. 1908 druckte das Blatt auf seiner Titelseite eine Karikatur des französischen Zeichners Lucien Haye (1876-1940), Abbildung 56.

Sie trägt den Titel Pejzažna okrajne stolicy (Landschaft am Rande der Hauptstadt) und zeigt einen Mann und eine Frau, die aus dem Fenster auf einen Bretterzaun blicken, hinter dem sich ein Fabrikgelände befindet. In der Bildmitte streicht ein Mann den Zaun mit grüner Farbe an. Die beiden Personen im Vordergrund kommentieren, welches Glück sie hätten, dass der Zaun grün gestrichen werde. So könnten sie denken, sie seien auf der Datscha. Den Hintergrund der Szene füllt die Fabrik, deren Schornsteine so stark rauchen, dass der gesamte Himmel verdunkelt ist. Die Redakteure wiesen ihre Leser nicht auf den französischen Ursprung der Zeichnung hin, sondern schrieben sie mit der Verwendung des Wortes dača (Datscha) in den russischen Kontext

122 Ruud: Fighting Words, S. 207, Polnoe sobranie zakonov Rossijskoj imperii, Tom 26, 1906, čast' 1, zakon No. 27574, S. 281-283, 18.03.1906 Ob" izmenenii i dopolnenii vremennych" pravil" o periodičeskoj pečati. Polnoe sobranie zakonov Rossijskoj imperii, Tom 26, 1906, čast' 1, zakon No. 27815, S. 481-483, 26.04.1906 O vremennych pravilach dlja nepovremennoj pečati. Zur Entwicklung der russischen Presse in den Revolutionsjahren 1905/06 bis zur Aufhebung der Zensur: Machonina: Istorija russkoj žurnalistiki načala XX veka, S. 17-25.

123 Hogarth: The Artist as Reporter, S. 101.

124 Für diese Analyse wurden die Karikaturenzeitschrift Budil'nik (Der Wecker), die von 1865-1917 in St. Petersburg erschien, ausgewertet (nur 1872 erschien die Zeitschrift nicht). Aufgrund ihrer kritischen Inhalte geriet die Zeitschrift besonders in den 186oer und 1870er Jahren immer wieder in Konflikt mit der Zensur. Mochnačeva (Hrsg.): Russkij illjustrirovannyj žurnal, S. 55-59.

125 Beispielsweise: o. A.: Uspechi russkoj promyšlennosti, in: Budil'nik, 24.06.1907, S. 1.

126 Beispielsweise: o. A.: Fabrika „Gor'kovskich geroev“ na Chitrovom rynke, in: Budil'nik, 19.09.1904, S. 2.

127 Beispielsweise: o. A.: „Otmyvanie“ Bolgi, in: Budil'nik, 15.09.1902, S. 1. 


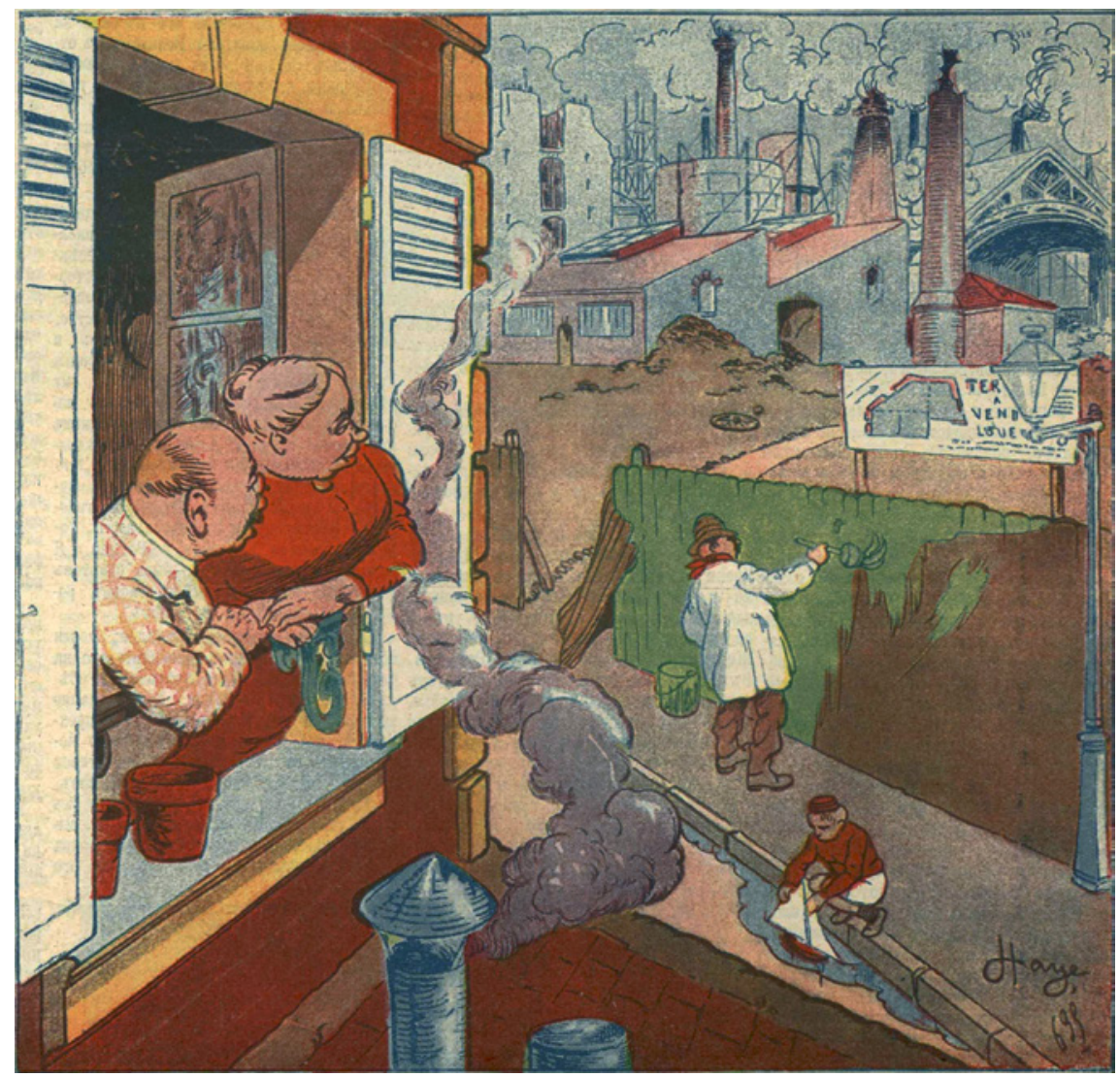

Abb. 56

Lucien Haye: Landschaft am Rande der Hauptstadt (Pejzaž na okrajne stolicy), in: Novoe Vremja, 23.02.1908, S. 1

ein. Die angesprochenen Probleme sollten also explizit nicht als ausländische Angelegenheit wahrgenommen werden.

Im Jahr 1908 war den Menschen im Zarenreich sehr wohl bewusst, dass Rauch und Abgase der Industrie gesundheitsschädlich waren. ${ }^{128}$ Arbeiterautoren beklagten immer wieder, dass Fabriken ihre gesamte Umgebung

128 Anlässlich der Hygieneausstellung in St. Petersburg zeigte ein Plakat im unteren Teil des Bildes einen kranken Mann, den die griechische Götting Hygieia am Arm fasst und der Sonne entgegenzieht. Der Grund für seine Krankheit ist unten links im Bild zu sehen, wo Fabrik- und Hafenanlagen mit schwarzem Rauch die Luft verpesten. Hier zeigt der Künstler sehr explizit die zerstörerische Kraft der Industrie. L. Ju. Vol'dman; Ju. A. Nikitin: Vystavočnaja Moskva. Exhibition of Moscow, Moskva 2006, S. 35, Abb. 1.38. Herzlichen Dank an Angelika Strobel für weitere Informationen zu diesem Plakat. 
verpesteten. ${ }^{129}$ Auch die zerstörerische Kraft, die Fabrikemissionen, Industrieabfälle und -abwasser auf die Natur hatten, registrierten die Zeitgenossen. Zwischen 1901 und 1903 erschienen in Budil'nik vier Karikaturen, die am Beispiel der Wolga auf die zunehmende Wasserverschmutzung durch Erdöl hinwiesen. ${ }^{130}$ Vor diesem Hintergrund dürfte der ironische Unterton und die kritische Haltung der Karikaturen gegenüber der Industrialisierung und der Verbreitung von Fabriken sowie der damit einhergehenden Folgen für Menschen und Natur für die Leser offensichtlich gewesen sein. ${ }^{131}$

Nach 1906 veröffentlichte zunächst Iskry besonders viele Fotografien von Arbeitslosen und Suppenküchen. Einrichtungen, die zu einem geringen Preis oder gratis Essen an Bedürftige ausgaben, waren in der russischen Hauptstadt schon vor der Revolution 1905/06 sehr verbreitet gewesen, doch erst jetzt erschienen Abbildungen in der Presse. ${ }^{132}$ Ab 1910 publizierte Ogonëk zahlreiche Beiträge über Wohltätigkeitsvereine. Ogonëk war mit zwei Rubeln für ein Jahresabonnement für die ärmeren Schichten erschwinglich ${ }^{133}$ und damit für diejenigen, die potentiell von vergleichbaren Einrichtungen profitierten. Doch auch die anderen Blätter setzten sich nach 1906 mit sozialen Problemen auseinander. ${ }^{134}$ Was in der russischen Presselandschaft aber fehlte, waren politisch linke illustrierte Zeitschriften, die Fotografien für ihre Argumentation einsetzten. ${ }^{135}$

Fotografische Aufnahmen in Illustrierten zeigten nicht die elenden Lebensumstände der Unterschicht. Stattdessen berichteten die Redakteure über Armutsbekämpfung und das Engagement wohltätiger Gesellschaften. ${ }^{136}$ Alle

129 Steinberg: Proletarian Imagination, S. 165.

130 Das Thema wurde in den Folgenden Ausgaben aufgegriffen: 21.10.1901, 15.09.1902, 28.09.1903, 02.11.1903. Zur Bedeutung der Wolga als Erinnerungs- und Identitätsstiftendem Ort: Guido Hausmann: Mütterchen Wolga. Ein Fluss als Erinnerungsort vom 16. bis ins frühe 20. Jahrhundert, Frankfurt a. M. 2009.

131 Ironie war um die Jahrhundertwende in der russischen Presse sehr verbreitet und bei den Lesern beliebt. Steinberg: Petersburg, S. 230-231. Weitere Karikaturen zu diesem Thema: Pėma: Približaetsja dačnyj sezon, in: Novoe Vremja, 05.04.1908, S. 1; Boba: Lono prirody, in: Novoe Vremja, 07.05.1911, S. 11; B. Amosovoj: O. T., in: Ogonëk, 03.09.1917, S. 1.

1321902 engagierten sich hier gut 390 Einrichtungen. V. N. Zanozina; E. A. Adamenko: Blagotvoritel'nost' i miloserdie. Rubeže XIX-XX vekov, Sankt-Peterburg 2010, S. 114.

133 Brooks: When Russia Learned to Read, S. 111-113.

134 Ogonëk veröffentlichte 14 Reportagen zu diesem Themenkomplex, Iskry neun, Niva fünf, Novoe Vremja drei und Ženskoe delo eine.

135 Beispielsweise druckten in London entsprechende Blätter Fotografien arbeitender Mütter und verglichen sie unter dem Stichwort des sozialen Imperialismus mit der Ausbeutung der Menschen in Afrika. Deborah Thom: Nice Girls and Rude Girls. Women Workers in World War I, London, New York 200o, S. 83, 85 .

136 Damit verfolgten die Redakteure eine ähnliche Strategie wie die Organisatoren der Pariser Salons: Aus Angst, den Betrachtern die traurige Realität der Hungerrevolten zuzumuten, 
Fotografien von Wohltätigkeitseinrichtungen zeigen viele Menschen. Abbildung $57^{137}$ beispielsweise zeigt im Vordergrund Kinder, die dick eingemummt Taschen, Suppenkannen und Töpfe tragen. Die Aufnahme stellt damit die Schwächsten der Gesellschaft ins Zentrum und betont dadurch die Notwenigkeit der Suppenküchen. ${ }^{138}$ Niva druckte ebenfalls 1906 eine seitenfüllende Fotokollage, die sich günstigen Wohnungen in St. Petersburg widmete und Aufnahmen von K. Bulla zeigte (Abb. 58).



Abb. 57

o. A.: Unentgeltliche Kantine in Petersburg. Im Haus erhalten die Armen gratis ein Mittagessen (Bezplatnyja stolovyja v Peterburge. Bednye polučivšie bezplatnye obedy na dome), in: Iskry, 27.08.19o6, S. 459

Die Aufnahmen auf der linken Seite zeigen eine Einzimmerwohnung mit integrierter Küche, auf der rechten Seite sind Gemeinschaftseinrichtungen wie Duschanlagen, eine Kantine und ein Klassenzimmer zu sehen. Die Unterkunft kostete neun Rubel im Monat und war auch für ärmere Familien

stellten sie Bilder von Wohltätigkeitsveranstaltungen aus. Dominique Lobstein: L'appel aux larmes. De la représentation des laissés-pour-compte de l'industrialisation dans la peinture des Salons officiels, 1879-1914, in: o. A. (Hrsg.): Des Plaines à l'usine. Images du travail dans la peinture française de 1870 à 1914, Paris 2001, S. 126-134, S. 127, 129.

137 CGAKFF f. 642.3, d. E 18954, Besplatnaja stolovaja u Putilovskogo zavoda, gruppa detej i vzroslych u vchoda stolovujo, Peterburg. In der Bildbeschriftung ist das Jahr 1913 genannt. Die Fotografie erschien allerdings ohne Nennung der Institution bereits 1906 in Iskry. o. A.: Bezplatnyja stolovyja v Peterburge. Bednye polučibšie bezplatnye obedy na dome, in: Iskry, 27.08.1906, S. 459 .

138 Die Aufnahme steht im Kontext von Diskussionen, die Journalisten in der russischen Presse um die Jahrhundertwende führten und die die Auswirkungen der Industrialisierung auf Kinder zum Thema hatten. Steinberg: Petersburg, S. 60. 


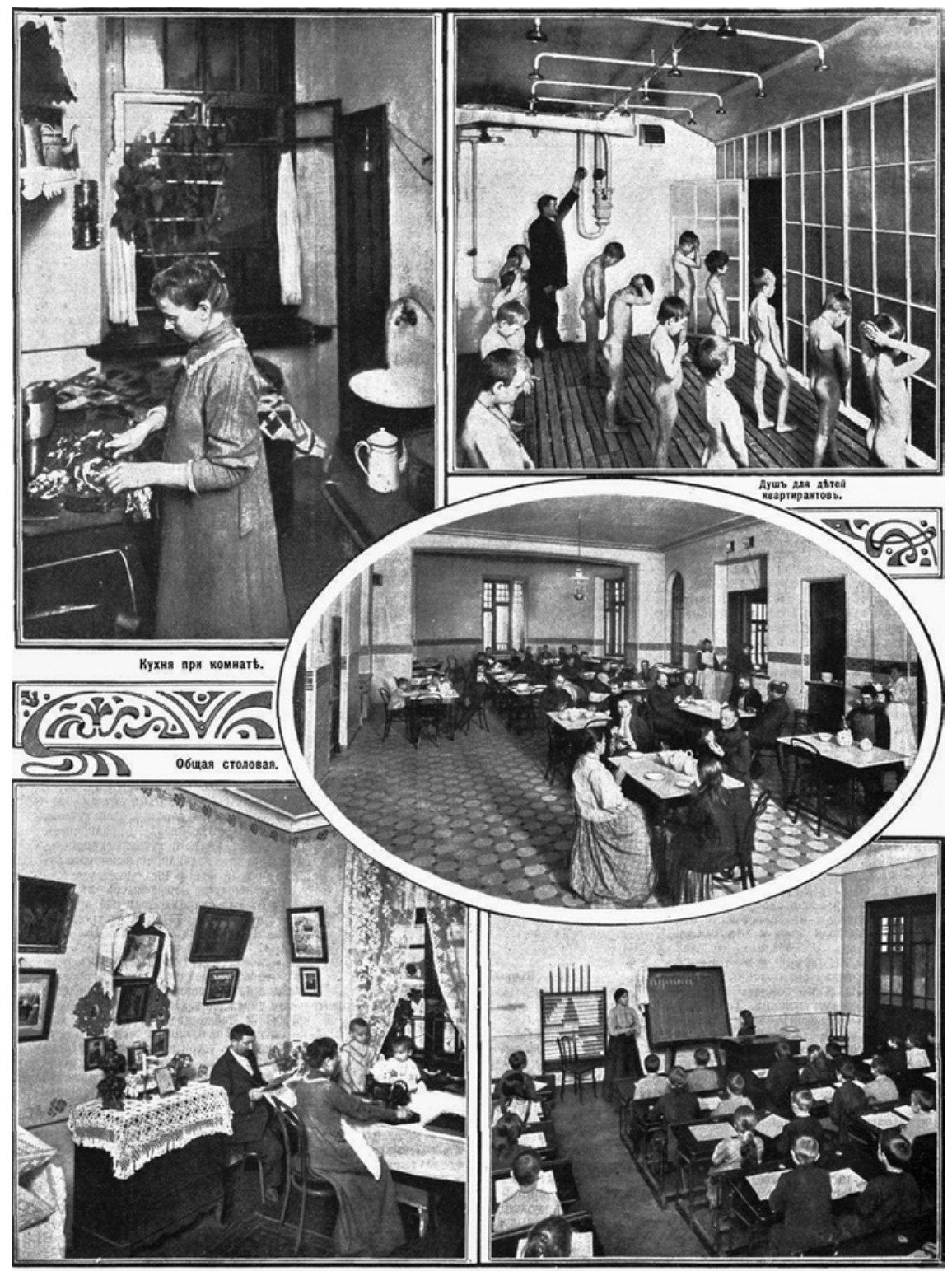

Комната, сдающаяся за 9 рүблей :ъ мtсящъ съ маленькой күхней

Швола дия дттеї.

Abb. $5^{8}$ Karl Karlovič Bulla: Günstiger Wohnraum in St. Petersburg (Deševyja žilišča v S.-Peterburge), in: Niva, 02.12.1906, S. 767 
erschwinglich. Allerdings vermitteln die Fotografien keinen Einblick in Wohnungen der Unterschicht, vielmehr scheint es sich um ein kleinbürgerliches Milieu zu handeln. Neben der Kleidung fällt auf der Aufnahme unten links die Nähmaschine auf, die von einer gewissen finanziellen Sicherheit zeugt. In der rechten oberen Ecke der Kollage ist eine Fotografie mit der Bildunterschrift „Dusche für die Kinder der Bewohner der Wohnungen“ zu sehen. Es wird suggeriert, dass auch Kinder aus ärmeren Schichten Zugang zu neuen hygienischen Errungenschaften hatten. ${ }^{139}$ Die Kollage entstand vor dem Hintergrund eines großen Wohnraummangels in der russischen Hauptstadt, der besonders die ärmsten Bevölkerungsschichten betraf. Dennoch muteten die Redakteure ihrem Publikum keine Fotografien der Unterschichten zu, sondern beschränkten sich auf ein repräsentables Milieu.

Die Illustrierten sparten nicht nur das Elend aus, sie schwiegen auch über die Gründe der katastrophalen hygienischen Situation in Moskau und St. Petersburg. Neben der unzureichenden Kanalisation bedrohten zunehmend Industrieabwässer die Trinkwasserqualität und damit die Gesundheit der Stadtbevölkerung. In den 189oer Jahren hatten sich beispielsweise am Ufer des Flusses Jauza in Moskau 120 Fabriken angesiedelt, darunter sechs Tuchfabriken und 24 Färbereien. Täglich flossen mehr als 583.00o Eimer Abwässer aus den Betrieben in das Gewässer. ${ }^{140}$ Trotz der offenkundigen Verschmutzung nutzen die Bewohner das Wasser der Newa und der Moskwa, um Wäsche zu waschen und zu baden. Außerdem speiste sich die Wasserversorgung der Metropolen teilweise ungefiltert aus dem Flusswasser, ungeachtet der Tatsache, dass sich Fabriken oberhalb der Wasserentnahme-Stationen befanden. ${ }^{141}$ Das verunreinigte Trinkwasser trug zur Verbreitung von Infektionskrankheiten wie Cholera und Ruhr bei. Es verwundert kaum, dass in Berichten russischer Illustrierter über Epidemien oder Tuberkulose, die als Arbeiterkrankheit galt, ${ }^{142}$

139 Eine ähnliche Fotografie aus dem Jahr 1902 findet sich im Bayer-Archiv Leverkusen. Die Fotografie zeigt männliche, nackte Arbeiter im Duschraum des Bayer Werks in Moskau. Allerdings ist unklar, in welchem Zusammenhang diese Fotografie entstanden ist, weil bei einer Neuverzeichnung innerhalb des Archivs Fotografien von ihren zugehörigen Dokumenten getrennt und gesondert im Bildarchiv archiviert wurden. Vermutlich ging es aber auch hier darum, die fortschrittlichen Sanitäranlagen zu demonstrieren und nicht unbekleidete Männer zu zeigen. BU: Objekt-Nr. 3435 .

140 Lee: Das Volk von Moskau und seine bedrohte Gesundheit, S. 34.

141 W. Jakowleff; G. Kulescha; K. Zabolotny; S. Zlatogoroff: Die Choleraepidemie von 1908/1909 in St. Petersburg, Veröffentlichungen aus dem Gebiete der Medizinalverwaltung, Bd. 2/3, Berlin 1913, S. 130.

142 Angelika Strobel: Die Gesundung Russlands. Hygienepropaganda in der Provinz um 1910, in: Jahrbücher für Geschichte Osteuropas, Jg. 61/2013, Heft 4, S. 531-551, S. 542; Michael Zdenek David: The White Plague in the Red Capital. The Control of Tuberculosis in Russia 
die Industrie nicht als mitverantwortlich auftauchte und die Autoren keine Verantwortlichen für die Probleme nannten.

Die einzige russische Illustrierte, in der ein Journalist in einem Artikel dezidiert Kritik gegenüber Unternehmern äußerte, war Ogonëk. Der zweiseitige Text behandelt das Leben der Bergleute im Donezbecken. ${ }^{143}$ Die Region hatte sich, nachdem der walisische Unternehmer John James Hughes (1815-1889) 1870 dort das erste Eisenhüttenwerk gegründet hatte, schnell zu einem Zentrum des Kohleabbaus und der Metallverarbeitung entwickelt. ${ }^{144}$ Das enorm schnelle Wachstum der Betriebe hatte zur Folge, dass sich die Bergwerks- und Hüttensiedlungen unkontrolliert und ohne Struktur ausbreiteten. Es fehlte an sanitären Einrichtungen, und die Siedlungen wurden zum Inbegriff schlechter Lebensbedingungen der russischen Industriearbeiter. ${ }^{145}$

Der Verfasser des Artikels schreckte nicht davor zurück, einzelne Unternehmer anzuprangern und ihnen vorzuwerfen, dass sie sich nicht um ihre Belegschaft und deren Lebensbedingungen kümmerten. Eine solche Bloßstellung von Missständen findet sich in keinem anderen Artikel. Der Fokus des Textes liegt auf dem alltäglichen Elend der einfachen Arbeiter. Der Journalist schildert im Fließtext das wilde Wachstum der Siedlungen und geht besonders auf die für die Region typischen Erdhütten ein, ${ }^{146}$ in denen die weniger gut ausgebildeten Arbeitskräfte lebten. Mittig auf der ersten Seite prangt eine Aufnahme des Fotografen G. F. Semeško, die dem Betrachter einen Überblick über eine Arbeitersiedlung gibt (Abb. 59).

Eine weitere Fotografie zeigt die Außenaufnahme einer Erdhütte. Semeško hatte die Region während der Wintermonate besucht, als der Schnee zu weiten Teilen den Morast verdeckte. Dadurch erwecken die Bilder einen relativ sauberen Eindruck. Erst die Bildunterschriften thematisieren Schmutz, fehlende Gärten und offene Kloaken zwischen den notdürftigen Unterkünften und vermitteln dem Betrachter ein Gefühl für die Zustände vor Ort. ${ }^{147}$

1900-1941, Chicago, Univ., Diss., 2007, S. 120; Friedrich Engels: Die Lage der arbeitenden Klasse in England, Nach eigner Anschauung und authentischen Quellen, in: Karl Marx; Friedrich Engels Werke, Bd. 2, Berlin (Ost) 1969, S. 225-506, S. 327.

143 o. A.: Kak živut šachtery-gornorabočie Doneckago bassejna, in: Ogonëk, 10.03.1913, S. 12-13.

144 Goehrke: Auf dem Weg in die Moderne, S. 299.

145 Hiroaki Kuromiya: Freedom and Terror in the Donbas. A Ukrainian-Russian Borderland, 1870s-1990s, Cambridge 1998, S. 17-18. Zu den Lebensbedingungen siehe auch: Charters Wynn: Workers, Strikes, and Pogroms. The Donbass-Dnepr Bend in Late Imperial Russia, 1870-1905, Princeton 1992, S. 34-35.

146 Theodore H. Friedgut: Iuzovka and Revolution, Bd. 1. Life and Work in Russia's Donbass, 1869-1924, Princeton 1989, S. 89-90.

147 Die Zustände im Donbass waren auch darum so prekär, weil sich beispielsweise in Juzovka die Unternehmer dafür einsetzten, dass die Siedlung trotz ihrer 48.ooo Einwohner im Jahr 




Abb. 59 G. F. Semeško: Wie leben die Bergarbeiter des Donezbeckens. Südrussische Gesellschaft für Steinkolebergbau (Kak živut' šachtery-gornorabočie Doneckago bassejna. Obščestvo južno-russkoj kamennougol'noj promyšlennosti), in: Ogonëk, 10.03 .1913 , S. 12

Im Vergleich zu den Außenaufnahmen verdeutlichen die Innenaufnahmen die beengten und dunklen Wohnverhältnisse. Abbildung 60 zeigt einen Wohnraum für 21 Arbeiter.

Auf dem Lichtbild sind lediglich neun von ihnen zu sehen. Dennoch war dem Fotografen Semeško daran gelegen, die beengten Wohnverhältnisse zu zeigen. Daraus resultierte die Bildkomposition, die drei auf dem Boden liegende Personen präsentiert, obwohl auf den Pritschen im Hintergrund genug Platz zu sein scheint, dass die Männer auch dort hätten schlafen können. Der Bildunterschrift zufolge musste sogar die Hälfte der Bewohner ihr Schlaflager auf dem Boden aufschlagen. Während diese Aufnahme noch relativ aufgeräumt und dadurch sauber wirkt, zeichnen die folgenden Fotografien ein dunkleres Bild (Abb. 61).

In beiden besetzt ein Herd oder Tisch das Zentrum der Illustration. Dadurch ist im Vordergrund weniger oder kein freier Fußboden sichtbar, was die Enge des Wohnraumes zusätzlich unterstreicht. Außerdem sind beide Abzüge sehr dunkel und illustrieren damit den Artikel, in dem es heißt, zwei Drittel der Zimmer seien sehr dunkel, und wo es dunkel sei, komme oft der Arzt. Auf allen drei Fotografien zeigt der starke Schattenwurf, dass Semeško mit Blitz arbeiten

1909 nicht den Status einer Stadt bekam. Dies hätte bedeutet, dass eine Stadtduma eingesetzt worden wäre, nach deren Vorgaben sich die Industriellen hätten richten müssen. Goehrke: Auf dem Weg in die Moderne, S. 299. 


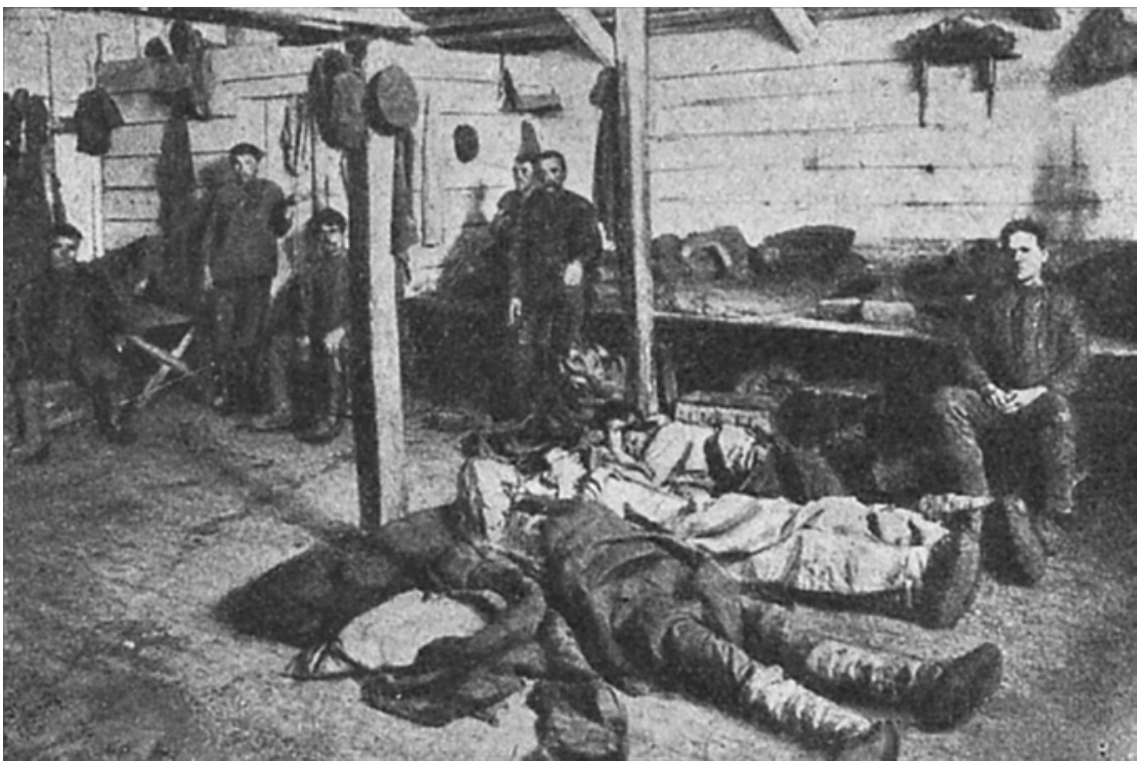

Abb. 60

G. F. Semeško: Wie leben die Bergarbeiter des Donezbeckens. Novorossijsker Gesellschaft des Schachts „Vetka“ (Kak živut' šachtery-gornorabočie Doneckago bassejna. Novorossijskoe obščestvo, Šachta „Vetka“), in: Ogonëk, 10.03.1913, S. 12

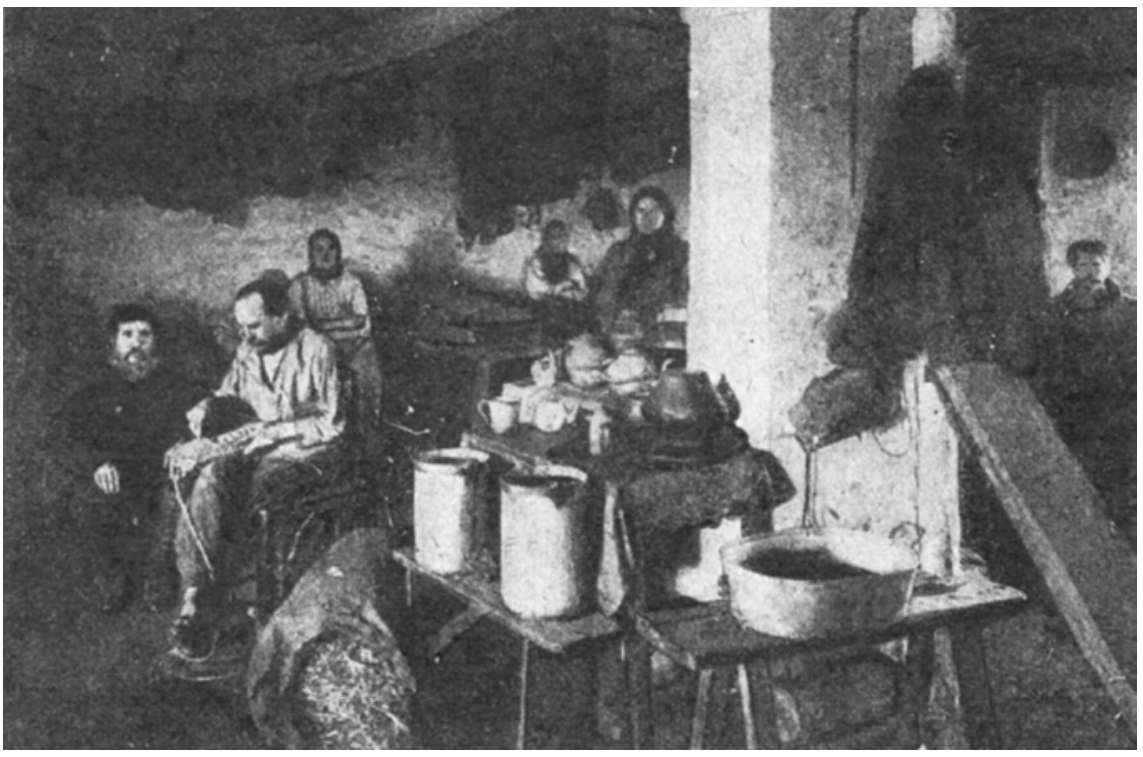

Abb. 61 G. F. Semeško: Wie leben die Bergarbeiter des Donezbeckens. Rutčenkovsker Steinkolegruben Gesellschaft (Kak živut' šachtery-gornorabočie Doneckago bassejna. Rutčenkovskoe tovariščestvo kamennougol'nych kopej), in: Ogonëk, 10.03.1913, S. 12 


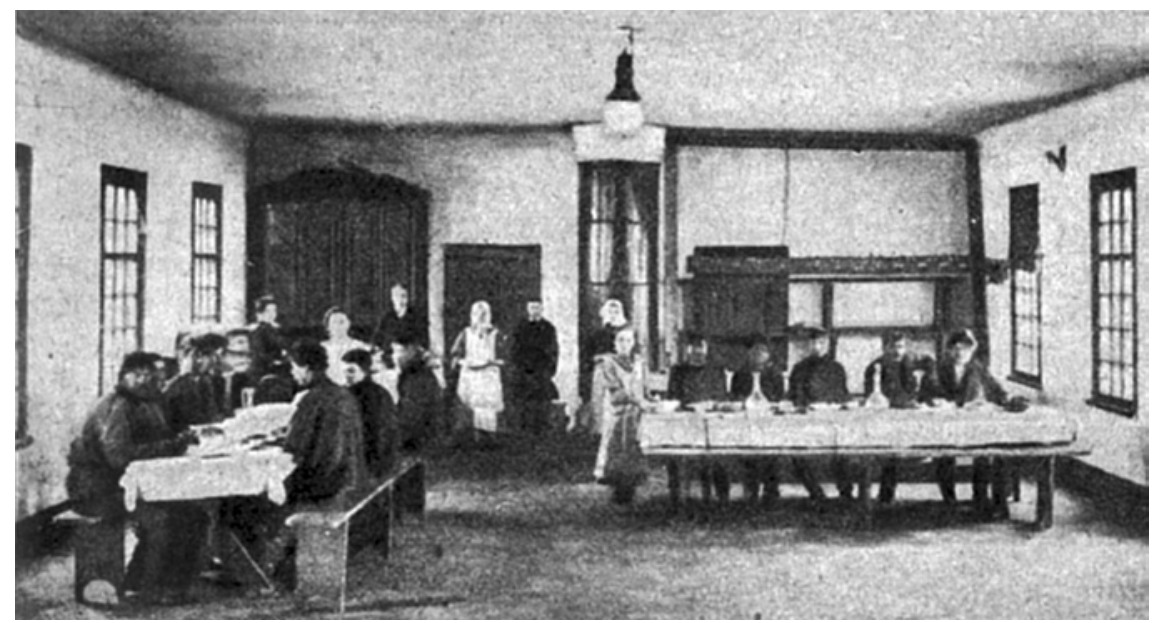

Abb. 62

G. F. Semeško: Wie leben die Bergarbeiter des Donezbeckens. Novorossijsker Gesellschaft des Schachts „Vetka“. Kantine für die Arbeiter (Kak živut' šachterygornorabočie Doneckago bassejna. Novorossijskoe obščestvo. Šachta „Vetka“, Stolovaja dlja rabočich), in: Ogonëk, 10.03.1913, S. 13

musste, um die Aufnahmen anfertigen zu können. Oftmals war in den Souterrainbehausungen die Türe die einzige Lichtquelle. ${ }^{148}$

Um die Missstände eindrücklich herauszuarbeiten verglich der Autor die Unterkünfte der ausländischen Arbeitskräfte und Meister mit denjenigen der einfachen russischen Bergleute. Diese Struktur übernahm Semeško auch für seine Aufnahmen. Die Wirkung der überbevölkerten, dreckigen und dunklen Innenräume stärkte er durch die Gegenüberstellung mit einer Fotografie aus einem Speisesaal für Ausländer und Meister (Abb. 62) und einer Außenaufnahme von deren Wohnhäusern. Letztere erinnern an westeuropäische Musterwohnungen für Arbeiter. ${ }^{149}$ Besonders die Fotografie der Kantine (Abb. 62) fungiert in der Reportage als Kontrast. Die Arbeiter sitzen auf Bänken an zwei Tischen, die parallel zur linken beziehungsweise zur rückwärtigen Wand des Raumes stehen. Damit bestimmen klare Geraden die Bildkonstruktion. Der leere Fußboden im Vordergrund, die weißen Tischdecken sowie die hellen Schürzen der Personen im Hintergrund betonen die Sauberkeit. Die Fenster an beiden Seiten des Raumes und die Lampe, die mittig von der Decke hängt,

148 Friedgut: Iuzovka and Revolution, S. 9o.

149 Jonas: Mulhouse et ses cites ouvrières, S. 66-212. 
können als Merkmale für ein gut durchlüftetes und damit im Verständnis der Zeitgenossen gesundes Umfeld gedeutet werden. ${ }^{150}$

Diese Reportage mit ihrer deutlichen Anklage und den Bildern aus den Behausungen der Arbeiter ist ein Einzelfall in den illustrierten Zeitschriften. Sie zeigt keine „Wunschbilder städtischer Eliten“, ${ }^{151}$ sondern das Elend der Arbeiter im Donbass. Dies war möglich, weil das Gebiet einerseits weit von der Hauptstadt entfernt war und somit fast einen exotischen Beigeschmack erhielt. Andererseits waren die Zustände in den dortigen Bergarbeitersiedlungen so unvorstellbar, dass Moskau und St. Petersburg im Vergleich geradezu als fortschrittliche Vorzeigestädte erscheinen mussten.

\subsection{Multiplikatoren des Konsums}

Während der Leser einer russischen Illustrierten im redaktionellen Teil wenig über die Industrialisierung und ihre Folgen erfuhr, fand er im Werbeteil zahlreiche Artikel, deren Autoren in einem eindeutig positiven, wenn nicht überschwänglichen Tonfall über Unternehmen berichteten. Haupt- und Anzeigenteil waren oft nur durch eine schmale, leicht zu übersehende Zwischenüberschrift oder grafische Elemente voneinander getrennt. ${ }^{152}$ Bis zum Ausbruch des Ersten Weltkriegs waren ca. 4o Prozent aller Artikel über Fabriken Reklame und erschienen folglich im Auftrag der Firmen. Davon druckten die Illustrierten die überwiegende Mehrzahl zwischen 1910 und 1913 - in einer Periode, in der die russische Wirtschaft einen Wachstumsschub erlebte und in der die Werbetätigkeit allgemein stark zunahm. ${ }^{153}$ Dies verdeutlicht, dass den Unternehmern viel daran lag, das Bild ihrer Betriebe in der Öffentlichkeit positiv darzustellen und für die eigenen Produkte zu werben.

Die Initiative für das Schalten einer Anzeige konnte sowohl von Fabrikanten als auch von Zeitschriften ausgehen. Industrielle wandten sich an Zeitungsund Zeitschriftenredakteure und schickten Annoncen zur Publikation an die Redaktionen. ${ }^{54}$ Der Preis hing sowohl von der Auflagenzahl als auch vom

150 Mediziner erstellten genaue Berechnungen, wieviel Kubikmeter Luft und wie viel Licht eine Person für ein gesundes Leben benötigte. Ben Eklof: Kindertempel or Shack? The School Building in Late Imperial Russia (A Case Study of Backwardness), in: Russian Review, Jg. 47/1988, Heft 2, S. 117-143, S. 122.

151 Raab: Die Feuerwehr und der Fotograf, S. 350.

$15^{2}$ Auch in russischen Zeitungen waren die Grenzen zwischen Werbung und redaktionellen Texten oft nicht deutlich. West: I Shop in Moscow, S. 3.

153 West: I Shop in Moscow, S. 14.

154 CGA Moskvy f. 318, op. I t 1, d. 1594, l. 31-32. 
Umfang der Anzeige und deren Platzierung innerhalb des Reklameteils ab. ${ }^{155}$ In anderen Fällen waren es die Zeitschriften selbst, die an die Fabrikanten schrieben und ihnen vorschlugen, in ihrer Publikation zu inserieren. So hatte der Kolomenskij mašinostroitel'nyj zavod am 12. Februar 1911 in der Zeitschrift Ogonëk eine Anzeige mit der Fotografie einer Lokomotive schalten lassen. Am 1. Januar 1912 kontaktierte der Herausgeber der Zeitschrift das Unternehmen. Er schilderte die Erfolge der Zeitschrift, betonte, dass diese die Auflage hatte erhöhen können und dass dies auch 1912 zu erwarten sei, und fragte, ob das Unternehmen vor diesem Hintergrund Interesse an einer erneuten Annonce habe. ${ }^{156} \mathrm{Ab} 1910$ machten die Einnahmen aus der Reklametätigkeit für die russischen Zeitungen bis zu einem Drittel der Gesamteinnahmen aus. ${ }^{157}$

Für ihre Annoncen gaben die Betriebe präzise Angaben zu Schrift und Layout vor ${ }^{158}$ und stimmten Form und Inhalt auf die zu erwartende Leserschaft ab. ${ }^{159}$ Im Vergleich zu Zeitschriften illustrierte das Fachjournal Zapiski imperatorskago russkago techničeskago ob̌̌čestva (Schriften der kaiserlichen russischen technischen Gesellschaft) seinen Werbeteil fast ausschließlich mit Druckgrafiken. ${ }^{160}$ Die Fabrikanten passten sich dem Erscheinungsbild des Journals an, das insgesamt nur wenige Fotografien druckte. Die Fachpublikation richtete sich an Ingenieure, die den größten Teil der Leserschaft stellten. Für sie waren technische Zeichnungen oft informativer als Fotografien entsprechender Maschinen, denn Grafiken konnten technische Details klarer darstellen als Autotypien.

Die einzige Ausnahme bei dieser Vorgehensweise war die Firma Siemens, die besonders aufwendige Reklamebeilagen veröffentlichen ließ. Es handelte

155 Beispielsweise kostete in der Zeitschrift Obščestvennyj vrač, die in einer Auflage von 3.00o Stück erschien, eine Anzeige im Umschlag des Blattes 30 Rubel, eine Halbeseite 15 Rubel und eine Viertelseite acht Rubel. In der restlichen Zeitschrift kostete Reklame für eine Seite 20 Rubel, eine Halbeseite zehn Rubel und eine Viertelseite fünf Rubel. Wurde die Anzeige mehrfach abgedruckt, bot das Blatt Reduktionen von 25 Prozent bei zehnmaligem und 20 Prozent bei fünfmaligem Abdruck an. CGA Moskvy f. 2105, op. 1, d. 345, l. $32-320$.

156 CGA Moskvy f. 318, op. 1, d. 1308, 1. 202. In diesem Fall sah die Fabrik jedoch von der erneuten Publikation einer Anzeige ab.

157 Dass die Werbeeinnahmen eine so große Bedeutung für die Redaktionen hatten, konnte sich erst nach den 186oer Jahren herausbilden. Im Zuge der Großen Reformen trat der russische Staat nämlich sein Privileg auf die Publikation von Werbung an die Herausgeber der Zeitungen und Zeitschriften ab. West: I Shop in Moscow, S. 31, 33; Häfner: Medienpräsenz, S. $275^{-276}$.

158 CGA Moskvy f. 2105, op. 1, d. 345, l. 39-40, 53, 101.

159 Damit orientierte sich Siemens an Handreichungen in zeitgenössischen Reklameratgebern. Chojnovskij (Hrsg.): Uspešnaja reklama, S. 26, 31.

16o Für diese Analyse wurde die gesamte Auflage der Zeitschrift von 1898 bis 1907 ausgewertet. 
sich um querformatige DINA4 Einlegeseiten, die einmal eingeklappt einer Halbseite der Zeitschrift entsprachen und immer ganz an deren Ende eingebunden waren (Abb. 63).

Die Firma orientierte sich damit an der zeitgenössischen Ratgeberliteratur zu Reklame, deren Autoren empfahlen, immer an der gleichen Stelle innerhalb der Publikation zu inserieren. Dies habe den Vorteil, dass sich das Schriftbild der Anzeige ins Unterbewusstsein der Leser einpräge und diese, noch bevor sie die eigentliche Seite aufschlügen, schon die Annonce vor dem inneren Augen sähen. ${ }^{161}$ Charakteristisch für das Aussehen der Reklameseiten von Siemens und Halske war neben dem Firmenschriftzug die grafische Rahmung der Anzeigen. Sie konnte wie auf Abbildung 63 relativ einfach ausfallen oder aus aufwendigen floralen Motiven bestehen. Die erste und die letzte Seite einer Publikation galten als besonders gute Position für eine Anzeige. ${ }^{162}$ Schrift und Bilder der Inserate der Firma Siemens erschienen im Vergleich zur Zeitschrift um 9o Grad gedreht, was die Aufmerksamkeit der Leser zusätzlich steigerte. ${ }^{163}$ Siemens präsentierte auf seinen Reklameseiten sowohl technische Zeichnungen, erklärende Begleittexte als auch hochwertige Fotografien. Während die deutsche Niederlassung von Siemens, wie es der Firmeninhaber forderte, ${ }^{164}$ fast keine Werbung machte und stattdessen ihre Produkte für sich werben ließ, unterhielten die russischen Niederlassungen eine rege Werbetätigkeit und fielen durch besonders innovative Designs auf. Diese Beilagen brachen mit dem sonst von Ingenieuren bevorzugten Medium der Zeichnung, und Siemens konnte sich mit seinen Anzeigen von der Konkurrenz abheben.

Im Gegensatz zu Fachjournalen druckten russische Illustrierte im Werbeteil neben einfachen Textannoncen mit Druckgrafiken auch mit Fotografien illustrierte Beiträge. Teilweise gaben die Autoren ihren Anzeigen den Anschein von Reportagen und verschleierten damit den Werbecharakter der Veröffentlichungen. ${ }^{165}$ Sie platzieren den Namen der Firma, ohne dass die Leser sofort realisierten, um welche Textform es sich handelte. ${ }^{166}$ Die Texte warben mit der modernen Ausstattung der Betriebe und den guten Arbeitsbedingungen. ${ }^{167}$

161 Ratner: Technika reklamy, S. 11.

162 Chojnovskij (Hrsg.): Uspešnaja reklama, S. 1o. Entsprechend mussten die Fabriken höhere Preise bezahlen, wenn sie ihre Anzeigen auf einer der beiden Seiten positionieren wollten. Beispielsweise: CGA Moskvy f. 318, op. 1, d. 1091, l. 77.

163 Holzer: Rasende Reporter, S. 70.

164 Sigfrid von Weiher: Werner von Siemens. Ein Leben für Wissenschaft, Technik und Wirtschaft, Göttingen u. a. 1970, S. 72.

165 Beispielsweise: Žak: Fabrika Gil'z „A. Katyk i Ko“ in: Novoe Vremja, 14.08.1910, S. 11.

166 Gerade Ende des 19. Jahrhunderts begannen Unternehmen immer stärker ihre Namen als Marken zu etablieren. West: I Shop in Moscow, S. 30.

167 Beispielsweise: Kokosovoe maslo „Žarovar“, in: Ogonëk, 12.02.1911, S. 18. 


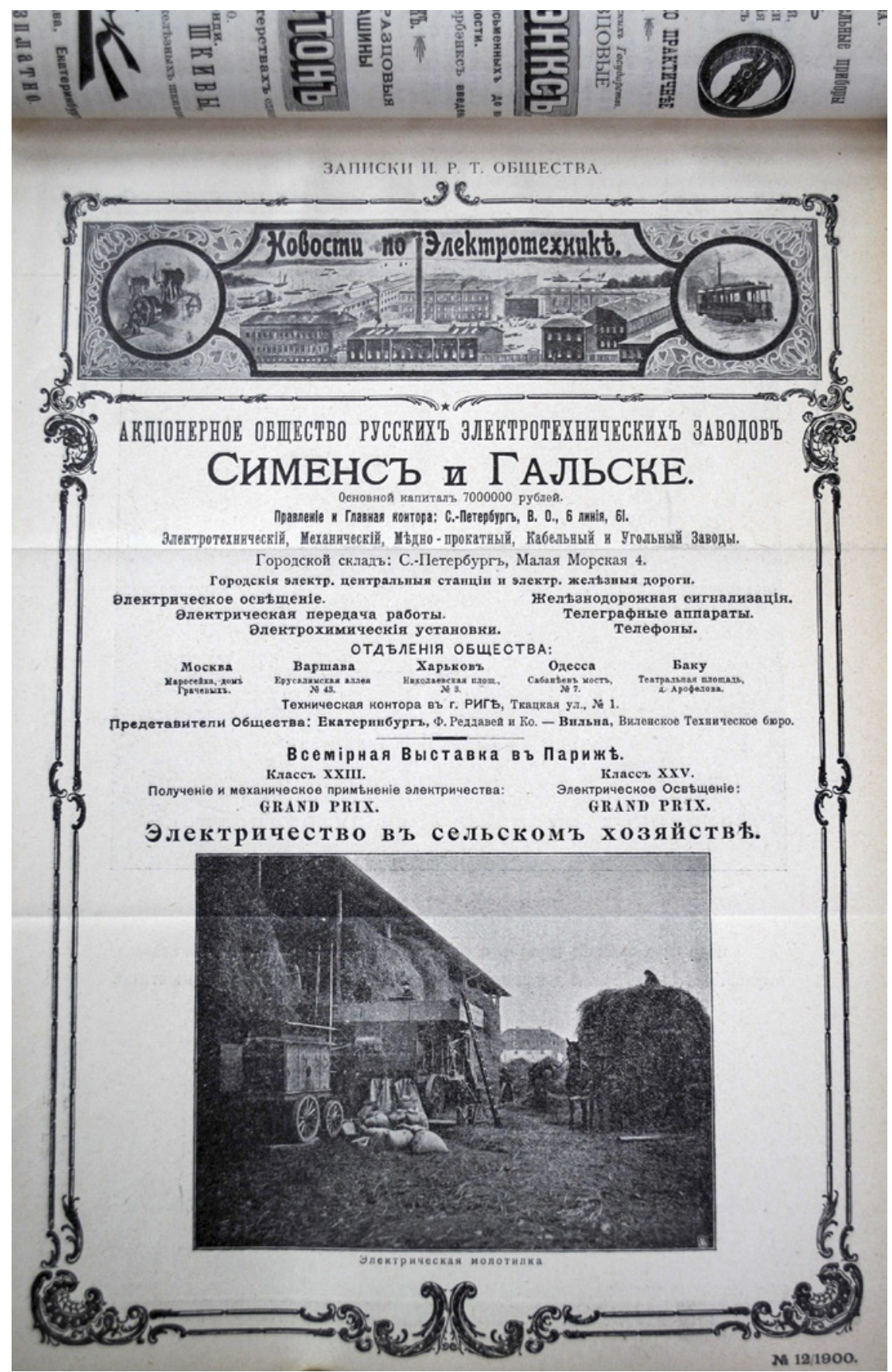

Abb. 63 o. A.: Neuigkeiten der Elektrotechnik (Novosti po èlektrotechniki), in: Schriften der Kaiserlich Russländischen Technischen Gesellschaft (Zapiski imperatorskogo russkago techničeskago obščestva), 1900, Heft 12, o. S. 
Produzenten von Seifen und Hygieneartikeln vermarkteten ihre Produkte gerne als Beitrag zum Wohle der gesamten Gesellschaft. ${ }^{168}$

Firmen aus der Nahrungsmittel-und Luxusgüterindustrie warben besonders häufig mit fotografisch bebilderten Artikeln. Die metallverarbeitende Industrie inserierte dagegen nur vereinzelt. ${ }^{169} \mathrm{Im}$ Gegensatz zu Schokolade und Seife waren Lokomotiven, Waggons oder Maschinen keine Produkte, die der durchschnittliche Zeitschriftenleser gekauft hätte. Wie die Analyse zeigt, war den Unternehmern sehr genau bewusst, welches Publikum sie über welches Blatt erreichten und wie sie ihre Reklame entsprechend anpassen mussten. So erschien in der hochpreisigen und eher luxuriös ausgestatteten Solnce Rossii im gesamten Untersuchungszeitraum nur eine Werbereportage, diese berichtete über die Produktion einer Süßwarenfabrik. 170

Die in den Werbeartikeln verwendeten Fotografien unterscheiden sich in ihrer Bildsprache nicht wesentlich von Industriefotografien aus Fotoalben. Zentrale Momente sind auch hier die Zentralperspektive und die Ästhetisierung der Fabrikräume. Zu diesem Zweck inszenierten Fotografen die geometrischen Elemente der Architektur und der Maschinen (Abb. 64). ${ }^{171}$

Die Unternehmer versuchten, die Leser mit Fotografien anzusprechen, die Fabriken als geordnete, überschaubare Räume zu zeigen, in denen der Mensch nicht vollständig von Maschinen dominiert wird, sondern Handarbeit nach wie vor einen wichtigen Anteil am Produktionsprozess hat. ${ }^{172}$ Im Vergleich zu den Artikeln im redaktionellen Teil der Zeitschriften fällt jedoch auf, dass so gut wie keine Bildunterschrift den Fotografen nennt. Dies könnte damit zusammenhängen, dass die Firmen die Fotografien in Auftrag gegeben hatten, die Rechte an den Bildern besaßen und darum kein Interesse hatten, weitere Namen aufzuführen. Denkbar wäre auch, dass die Fotografen selbst keinen Wert darauf legten, in diesem Kontext in Erscheinung zu treten.

Während die Fotografien der Nahrungs- und Genussmittelindustrie eher den Produktionsablauf zeigen, stellen die wenigen Beiträge aus der metallverarbeitenden Industrie das fertige Produkt ins Zentrum des Lichtbildes.

168 Ähnliche Strategien finden sich auch in Annoncen russischer Zeitungen. West: I Shop in Moscow, S. 99, 101.

169 Während Firmen aus der Nahrungs- und Luxusgüterindustrie 19 Annoncen schalteten, gaben Betriebe der metallverarbeitenden Industrie nur fünf in Auftrag.

170 Bligken i Robinson, in: Solnce Rossii, 09.09.1912, Anhang.

171 Kapitel „Neue Bilder eines neuen Raums - Fabrikalben“, S. 103-178.

172 Dies änderte sich in der frühen Sowjetunion. Ende der 1920er Jahre zeigten Fotografien auch schmutzige Arbeiter, und Fabriken wurden als Schlachtfelder inszeniert, in denen für die neue Gesellschaft gekämpft wurde. Tupitsyn: The Soviet Photograph, S. 74-77, 120. 


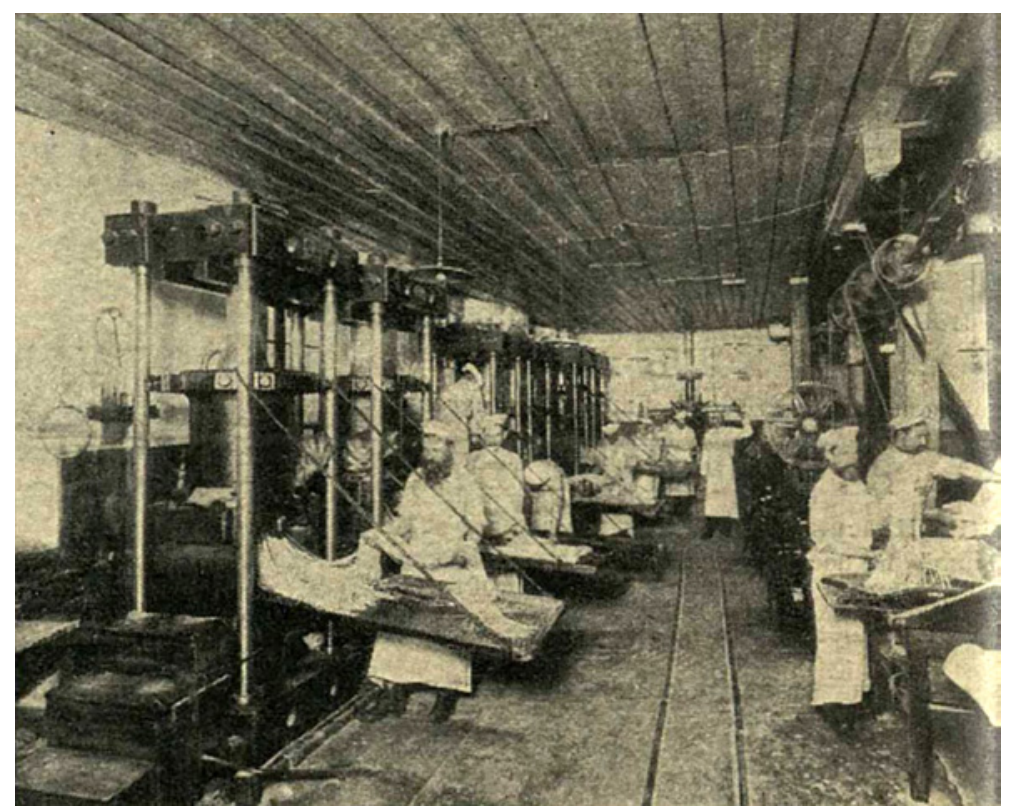

Abb. 64 o. A.: Fabrik Bligken und Robinzon. Nudelabteilung (Fabrika Bligken i Robinzon. Makaronnoe otdelenie), in: Novoe Vremja, 29.05.1910, S. 12

Fotografien von Hochöfen oder andere Ansichten, in denen die Monumentalität der neuen Technik besonders betont wird, fehlen. ${ }^{173}$ Stattdessen ergänzten Fotografien von Lokomotiven, Waggons, Maschinen oder Brücken die Texte. ${ }^{174}$ Diese Aufnahmen wirken zum überwiegenden Teil statisch, sie zeigen weder Dampfwolken noch den Funkenregen, und wie er entstand, wenn in die Stahlschmelze Sauerstoff geblasen wurde. Im Gegensatz zu Deutschland ${ }^{175}$ zeigten russische Zeitschriften keine Faszination für Stärke und Wucht der Technik.

Die einzigen Abbildungen, die die Monumentalität der modernen Industrie einzufangen suchten, waren Reproduktionen von Gemälden. Niva publizierte 1900 eine Kopie des „Eisenwalzwerk" des deutschen Malers Adolph von

173 Beispielsweise galten neben dem Stahlabstich Dampfhämmer als Inbegriff der neuen Technik. Grassias: Les Grandes Usines de Turgan, S. 37; Jürgen Hannig: Fotografie als historische Quelle, in: Klaus Tenfelde (Hrsg.): Bilder von Krupp. Fotografie und Geschichte im Industriezeitalter, München 1994, S. 269-287, S. 273-287.

174 Beispielsweise: Kolomenskij mašinostroitel'nyj zavod, in: Ogonëk, 12.02.1911, S. 19; Akkumuljatornyj vagon-samochod Brjanskogo zavoda, in: Ogonëk, 01.01.1911, S. 27; Russkoe obščestvo mašinostroitel'nych zavodov Hartman, in: Ogonëk, 25.12.1912, S. 23.

175 Lüdtke: Industriebilder - Bilder der Industriearbeit?, S. 413-414. 
Menzel aus dem Jahr 1872/1875. Das Bild stellt bis heute eine der Ikonen nicht nur deutscher Industriemalerei dar und besticht aufgrund der Dynamik, die Menzel eingefangen hatte, sowie durch die Detailgenauigkeit. Es verfügt über einen breiten Interpretationsspielraum, und sowohl Befürworter als auch Kritiker der Industrialisierung vereinnahmten es für ihre Argumentation. ${ }^{176}$ Ein weiteres Gemälde, das Niva abdruckte, war „Lokomotivbau bei Borsig“ (1874) von Paul Friedrich Meyerheim (1842-1915) ${ }^{177}$ Hier verdeutlicht die Größe der Lokomotive gegenüber den Menschen die Kraft der neuen Technik. Im Mittelpunkt des Gemäldes steht eine Gruppe Ingenieure, deren großer, weißer Bauplan die Beherrschung der Technik durch den Menschen symbolisiert. Die menschliche Kontrolle der Naturkräfte mittels Maschinen war in den Fotografien in russischen Illustrierten kein Thema, weder in den wenigen Berichten im redaktionellen Teil noch in den Reklametexten und Bildern. Bei Illustrierten handelte es sich um Konsumprodukte, welche die Menschen in ihrer Freizeit unterhalten sollten. Zu realistische Fotografien wären vor diesem Hintergrund möglicherweise verstörend gewesen.

\subsection{Fazit}

Illustrierte hatten das Ziel, ihre Leser zu informieren und zu unterhalten. Aus diesem Grund legten die Herausgeber der Blätter einen Schwerpunkt auf kulturelle Themen wie Literatur, Theater und Musik. Je nach Zeitschrift ergänzten Berichte über Sport, Wissenschaft oder politische und gesellschaftliche Ereignisse das Themenspektrum. Abhängig davon, an welches Publikum sich die Blätter richteten, war Industrie mehr oder weniger oft Gegenstand von Artikeln. Solnce Rossii und Ženskoe delo griffen das Thema so gut wie nie auf, während Niva und Ogonëk häufiger über Fabriken berichteten. Auch wenn die Illustrierten selbst Kinder der Industrialisierung waren, spielte diese in ihnen nur eine marginale Rolle. Dies galt für positive wie negative Berichterstattung, denn bis auf wenige Ausnahmen stellten die Autoren Unternehmen weder besonders positiv dar, noch kritisierten sie sie offen. Wenn Zeitgenossen in Zeitschriften Kritik an den negativen Auswirkungen der Industrialisierung auf Menschen und Natur äußerten, nutzten sie überwiegend Kariakturen. Fotografien schienen nicht das geeignete Medium gewesen zu sein. Zeichnungen

176 Osterhammel: Verwandlung der Welt, S. 980; Türk: Bilder der Arbeit, S. 172-173.

177 Türk: Bilder der Arbeit, S. 174-175. Abgedruckt in: Niva, 22.09.1912, S. 761. 
ermöglichten, komplexe Sachverhalte zu verdichten und mittels Metaphern sichtbar zu machen.

Fotografien von Unternehmen erhielten dann einen Platz in den Zeitschriften, wenn Journalisten über sie in Zusammenhang mit Jubiläen und mit der Luft- oder Schifffahrt berichteten. Besonders beliebt waren beim russischen Publikum Beiträge über Katastrophen - ein weiterer Kontext, in dem Betriebe die Aufmerksamkeit der Journalisten bekamen. Allerdings waren diese Berichte in erster Linie auf die Sensation der Unglücke und nur sekundär auf die Fabriken ausgerichtet. Lichtbildner entwickelten eine Bildsprache, mit der sie versuchten, die neuen Zerstörungskräfte der Industrie einzufangen. Verbogene Stahlelemente entwickelten sich zu einem zentralen Element dieser Katastrophenfotografie.

Über soziale Probleme, die Folgen der Industrialisierung waren, berichteten Redakteure bis 1905 kaum. Nachdem die russische Regierung die Zensur der Presse 1906 weitgehend aufgehoben hatte, erschienen in den Illustrierten zwar Berichte über soziale Missstände, jedoch ohne dass Fotografien der prekären Lebensbedingungen gezeigt worden wären. Meist würdigten die Beiträge stattdessen das Engagement von Wohltätigkeitsorganisationen. Sie versicherten damit ihren Lesern, dass etwas gegen die Missstände unternommen werde. Die Herausgeber der Illustrierten hatten nicht das Ziel, breite Teile der russischen Gesellschaft zum sozialen Engagement und zur kritischen Auseinandersetzung mit den gesellschaftlichen Zuständen aufzurufen.

Für die Zeit vor der Aufhebung der Pressezensur ließe sich annehmen, dass es Journalisten schlicht nicht möglich war, über die prekären Lebensbedingungen der unterprivilegierten Schichten zu berichten. Die Tatsache, dass das Thema auch nach 1906 kaum direkt zur Sprache kam, zeigt aber, dass in den Redaktionen ebenfalls wenig Interesse an der Auseinandersetzung mit dieser Realität bestand. Dieses Desinteresse dürfte mit ökonomischen und verkaufsstrategischen Überlegunen zusammenhängen. Denkbar wäre auch, dass die gesellschaftlichen Eliten des Russischen Reichs zunehmend den Bezug zur einfachen Bevölkerung verloren. Sie registrierten die Thematik und ihre Bedeutung für die russische Gesellschaft nicht mehr. Dieser Realitätsverust rächte sich und es foglte ein ,böses Erwachen' in den revolutionären Auseinandersetzungen der Jahre 1905/06 und 1917.

Diese Marginalisierung in der Berichterstattung wollten Industrielle nicht tatenlos hinnehmen, weshalb sie sich aktiv dafür einsetzten, dass ihre Unternehmen in den Zeitschriften einen Platz erhielten. Die geeignete Plattform bot ihnen der Reklameteil, wo sie nach ihren eigenen Vorstellungen über ihre Produkte und Betriebe informieren konnten. Dabei passten sie sich an das 
Medium der Illustrierten an und ,tarnten' ihre Artikel häufig als Reportagen. Die zugehörigen Fotografien zeigen, ähnlich wie in den Fotoalben, die Fabriken als geordnete, klar strukturierte Räume. Die Unternehmer wählten für ihre Annoncen keine fotografischen Aufnahmen großer Maschinen, Hochöfen oder Eisenschmelzen, welche die gewaltigen Kräfte betont hätten, die in der Industrie am Werk waren. Es blieb Gemäldereproduktionen und Stichen vorbehalten, die schweißtreibende Tätigkeit der Arbeiter zu illustrieren. Auf diese Weise erhielt der Ort der Massenproduktion doch Zugang ins Konsumprodukt Illustrierte. 